ARTICLE

Received 7 Apr 2016 | Accepted 8 Nov 2016 | Published 22 Dec 2016

DOI: $10.1038 /$ ncomms13894

OPEN

\title{
Targeting dendritic cells to accelerate T-cell activation overcomes a bottleneck in tuberculosis vaccine efficacy
}

Kristin L. Griffiths ${ }^{1}$, Mushtaq Ahmed ${ }^{1}$, Shibali Das ${ }^{1}$, Radha Gopal ${ }^{2}$, William Horne ${ }^{2}$, Terry D. Connell ${ }^{3}$, Kelly D. Moynihan ${ }^{4}$, Jay K. Kolls ${ }^{2}$, Darrell J. Irvine ${ }^{5}$, Maxim N. Artyomov', Javier Rangel-Moreno ${ }^{7}$ \& Shabaana A. Khader ${ }^{1}$

The development of a tuberculosis (TB) vaccine that induces sterilizing immunity to Mycobacterium tuberculosis infection has been elusive. Absence of sterilizing immunity induced by TB vaccines may be due to delayed activation of mucosal dendritic cells (DCs), and subsequent delay in antigen presentation and activation of vaccine-induced CD4 + T-cell responses. Here we show that pulmonary delivery of activated $M$. tuberculosis antigen-primed DCs into vaccinated mice, at the time of $M$. tuberculosis exposure, can overcome the delay in accumulation of vaccine-induced CD4 ${ }^{+}$T-cell responses. In addition, activating endogenous host $\mathrm{CD}_{103}{ }^{+} \mathrm{DCs}$ and the CD40-CD40L pathway can similarly induce rapid accumulation of vaccine-induced lung CD4 ${ }^{+}$T-cell responses and limit early $M$. tuberculosis growth. Thus, our study provides proof of concept that targeting mucosal DCs can accelerate vaccineinduced T-cell responses on $M$. tuberculosis infection, and provide insights to overcome bottlenecks in TB vaccine efficacy.

\footnotetext{
${ }^{1}$ Department of Molecular Microbiology, Washington University in St Louis, 660 S Euclid Avenue, St Louis, Missouri 63110, USA. ${ }^{2}$ Richard King Mellon Foundation Institute for Pediatric Research, Children's Hospital of Pittsburgh of UPMC, 4401 Penn Avenue, Pittsburgh, Pennsylvania 15224, USA. ${ }^{3}$ Witebsky Center for Microbial Pathogenesis \& Immunology, Department of Microbiology \& Immunology, University at Buffalo, 3435 Main Street, Buffalo, New York 14214, USA. ${ }^{4}$ Department of Biological Engineering, Massachusetts Institute of Technology, 500 Main Street, Cambridge, Massachusetts 02139, USA. ${ }^{5}$ Department of Materials Science and Engineering, Massachusetts Institute of Technology, 77 Massachusetts Avenue, Cambridge, Massachusetts 02139 , USA. ${ }^{6}$ Division of Immunobiology, Washington University in St Louis, 660 S Euclid Avenue, St Louis, Missouri 63110, USA. ${ }^{7}$ Department of Medicine, Division of Allergy, Immunology and Rheumatology, University of Rochester Medical Center, 601 Elmwood Avenue, Rochester, New York 14642, USA. Correspondence and requests for materials should be addressed to S.A.K. (email: khader@wustl.edu).
} 
T uberculosis (TB) is a leading cause of death by infection ${ }^{1}$. TB is caused by aerosol exposure to the intracellular bacterium Mycobacterium tuberculosis (Mtb), leading to either latent disease or active pulmonary disease. The only currently licensed vaccine against TB is Mycobacterium bovis Bacille Calmette-Guerin (BCG). Although BCG vaccination is effective against childhood forms of $\mathrm{TB}^{1}$, and in decreasing childhood TB morbidity ${ }^{1}$, it provides variable efficacy against adult pulmonary TB. Thus, over the past two decades, concerted efforts have been made to develop new vaccines for TB that will provide improved protection on Mtb exposure. Modern candidate $\mathrm{TB}$ vaccines have focussed on induction of $\mathrm{T}$-cell responses, primarily $\mathrm{CD} 4^{+} \mathrm{T}$ cells producing interferon gamma $(\text { IFN } \gamma)^{2}$. More recently, an important role for mucosal interleukin 17A (IL-17A) in vaccine-induced protection against TB disease has been shown ${ }^{3-6}$. Thus, induction of lung-resident IL-17Aproducing $\mathrm{CD} 4{ }^{+} \mathrm{T}$-cell populations by $\mathrm{TB}$ vaccines is also being explored ${ }^{2,4,5,7}$. Despite these efforts, most TB vaccines reduce the burden of lung $M t b$ by $\sim 0.5-1.0 \log$ in animal challenge models $^{3,8-11}$. Recombinant live mycobacterial vaccines confer improved protection ( $\sim 2 \log$ reduction), when compared with subunit and virally vectored $\mathrm{TB}$ vaccines. Examples of recombinant vaccines include the recombinant Mycobacterium smegmatis vaccine, which induces sterilizing immunity in the liver, but not the lung ${ }^{12}$; recombinant $\mathrm{BCG}$ over-expressing Listeria monocytogenes listeriolysin and lacking urease $\mathrm{C}^{11,13}$; and the recombinant $M t b$ vaccine lacking $P h o P^{14}$. Other work has highlighted the benefit of administering recombinant $M t b$ vaccines mucosally, showing that macaques vaccinated with the attenuated $M t b$ mutant lacking $S i g H$ had sterile protection in some $\mathrm{TB}$ lesions ${ }^{15}$. Although these results are promising, considerable challenges are associated with the design and implementation of a recombinant $M t b$ vaccine to be delivered mucosally via the lungs, particularly in light of the TB-HIV co-epidemic. Thus, it is critical to fully understand the early events occurring in the vaccinated $M t b$-infected lung.

After $M t b$ infection of naive mice, accumulation of activated lung $\mathrm{CD} 4^{+} \mathrm{T}$ cells is delayed, occurring between 14 and 21 days post $M t b$ infection (dpi) ${ }^{16,17}$. This delay is thought to be due to $M t b$-mediated inhibition of early antigen presentation by antigenpresenting cells (APCs) ${ }^{18-20}$. Even in the presence of an existing $M t b$-specific vaccine-induced $\mathrm{CD} 4^{+} \mathrm{T}$-cell population in the lung, $\mathrm{CD} 4^{+} \mathrm{T}$-memory cells do not accumulate in the lung until $12-14 \mathrm{dpi}^{3,6,21}$. Priming of T-cell responses following primary $M t b$ infection requires trafficking of $M t b$-infected DCs from the lungs to the lymph nodes $(\mathrm{LNs})^{20,22}$. Furthermore, $M t b$-infected DCs do not efficiently present antigen directly to $M t b$-specific $\mathrm{CD} 4{ }^{+} \mathrm{T}$ cells, but antigen is transferred from $M t b$-infected DCs to uninfected bystander DCs in the LNs, for antigen presentation to naive $\mathrm{CD} 4{ }^{+} \mathrm{T}$ cells ${ }^{22}$. On $M t b$ infection, even vaccine-induced memory $\mathrm{T}$ cells accumulate in the LNs before mobilization to the lungs ${ }^{6}$. Thus, in the current study, we hypothesized that the delay in accumulation of lung vaccine-induced $\mathrm{CD} 4{ }^{+} \mathrm{T}$ cells in vaccinated $M t b$-infected hosts is due to a delay in antigen presentation by $M t b$-infected APCs, thus resulting in a bottleneck and preventing sterilizing immunity to $M t b$ infection. We show that we can overcome the delay in accumulation of vaccineinduced memory $\mathrm{CD} 4{ }^{+} \mathrm{T}$ cells by transferring exogenously primed activated DCs into the lungs of vaccinated mice at the time of $M t b$ infection. DC transfer into vaccinated $M t b$-infected hosts results in rapid activation of vaccine-induced $\mathrm{CD} 4{ }^{+} \mathrm{T}$-cell responses, substantial changes in the lung micro-environment, activation of lung alveolar macrophages and early $M t b$ control. Furthermore, these protective mechanisms are dependent on $\mathrm{CD}_{103}{ }^{+}$DCs and the CD40-CD40L activation pathway, as host-directed therapeutics targeting these pathways in vaccinated
$M t b$-infected mice can mimic the protective effects of pulmonary DC transfer. Thus, we have determined a key bottleneck for the failure of $\mathrm{TB}$ vaccines to induce sterilizing protection against $M t b$ infection. These results provide a roadmap for the type of immune responses that a sterilizing TB vaccine should induce, representing a milestone in our mechanistic understanding of $\mathrm{TB}$ vaccine-mediated immune responses.

\section{Results}

DC transfer confers superior vaccine-induced Mtb control. Following $M t b$ infection, vaccine-induced $\mathrm{CD}_{4}{ }^{+} \mathrm{T}$-cell responses are delayed in vaccinated hosts ${ }^{3,6}$, and could be a likely reason for the failure of $\mathrm{TB}$ vaccines to induce sterilizing immunity. Therefore, we first assessed whether a delay in accumulation of $\mathrm{CD} 4{ }^{+} \mathrm{T}$-cell recall responses was due to an inherent deficiency in the ability of the vaccine-induced $\mathrm{T}$ cells to respond to antigen exposure. Thus, naive $\mathrm{CD} 4^{+} \mathrm{T}$ cells were isolated from $M t b$-specific immunodominant antigen $85 \mathrm{~B}$ (Ag85B) T-cell receptor transgenic (TCR) mice and adoptively transferred into congenic C57BL/6 (B6) mice, which were vaccinated subcutaneously (s.c.) with BCG, rested for 4 weeks followed by a mucosal boost with the $\mathrm{CD} 4{ }^{+} \mathrm{T}$-cell epitope $\mathrm{Ag} 85 \mathrm{~B}_{240-254}$. Following a period of rest for 4 weeks, lung $\mathrm{CD} 44^{+} \mathrm{T}$ cells were isolated and cultured in vitro in the presence of Ag85B-pulsed DCs. Recall Ag85B-specific CD4 ${ }^{+} \mathrm{T}$ cells proliferated rapidly, underwent activation, produced cytokines and on co-culture with $M t b$-infected macrophages could mediate $M t b$ control (Fig. 1a-d). These data suggest that vaccine-induced $\mathrm{CD} 4{ }^{+}$ $\mathrm{T}$ cells can rapidly respond to $M t b$ antigen, but activation is delayed in vivo following $M t b$ infection $^{3,6,21}$. This is not due to a limitation of $M t b$ antigen availability, as infection of vaccinated mice with high doses ( $\sim 1,000$ c.f.u.) of $M t b$ did not improve vaccine-induced $M t b$ control (Supplementary Fig. 1a) ${ }^{23}$. $\mathrm{T}$ regulatory cells induced in response to $M t b$ infection are known to suppress early T-cell responses ${ }^{24}$. As before ${ }^{25}$, however, depletion of $\mathrm{T}$ regulatory cells in vaccinated $\mathrm{B} 6$ mice early following $M t b$ infection did not improve vaccine-induced $M t b$ control, instead increasing susceptibility to $M t b$ infection (Supplementary Fig. 1b). Thus, although recall $\mathrm{CD}^{+}{ }^{+} \mathrm{T}$-cell responses are delayed in vivo in $M t b$-infected hosts ${ }^{3,6,21}$, vaccineinduced $\mathrm{CD} 4^{+} \mathrm{T}$ cells can rapidly respond ex vivo to activate macrophages for $M t b$ control.

To overcome the delayed activation of $\mathrm{CD} 4{ }^{+}$T-cell recall responses on $M t b$ infection in vivo, we addressed whether delivery of $M t b$ antigen-pulsed DCs into the lungs of $M t b$-infected-vaccinated mice could overcome this bottleneck and improve recall vaccine-induced $\mathrm{CD} 4{ }^{+} \mathrm{T}$-cell immunity. B6 mice were thus vaccinated with BCG, rested for 4 weeks, followed by a mucosal boost with $A g 85 B_{240-254}$. Mice were further rested for 4 weeks, after which they were challenged with a clinical W-Beijing Mtb strain, HN878 (ref. 26). At the time of $M t b$ challenge, vaccinated mice received $\mathrm{Ag}_{85} \mathrm{~B}_{240-254}$-pulsed B6 bone marrow-derived DCs (BMDC) delivered intratracheally (i.t., DC transfer). While vaccination and mucosal boost reduced lung $M t b$ burden as before ${ }^{4}$, B6 DC transfer into vaccinated mice reduced lung $M t b$ burden by $\sim 98.6 \%$, when compared with the burden in unvaccinated $M t b$-infected mice (Supplementary Fig. 2a,b). Pulmonary delivery of $\mathrm{Ag}_{85} \mathrm{~B}_{240-254}$ peptide alone did not induce the superior protection seen with DC transfer in vaccinated mice (Supplementary Fig. 2a), further suggesting that antigen availability was not the major reason for delayed vaccineinduced $\mathrm{CD} 4{ }^{+} \mathrm{T}$-cell recall responses. To further improve the ability of antigen-pulsed DCs to activate $\mathrm{CD} 4^{+} \mathrm{T}$ cells following $M t b$ infection, we next stimulated DCs with the Dectin-1 agonist zymosan (Z-DC) ${ }^{27}$ at the time of antigen pulsing. Activating 
a

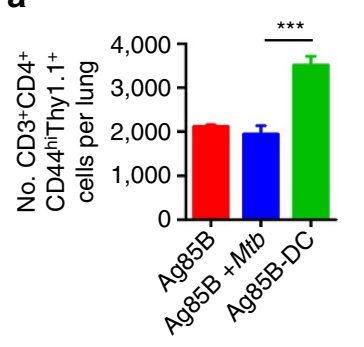

b

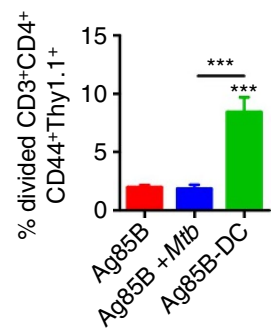

C

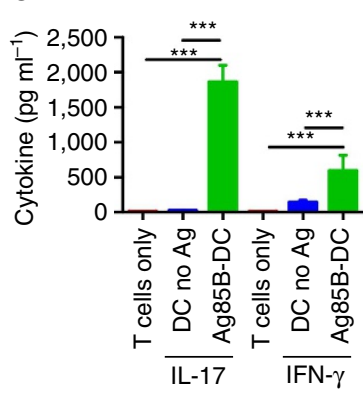

d

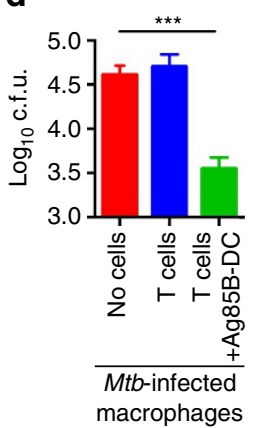

e

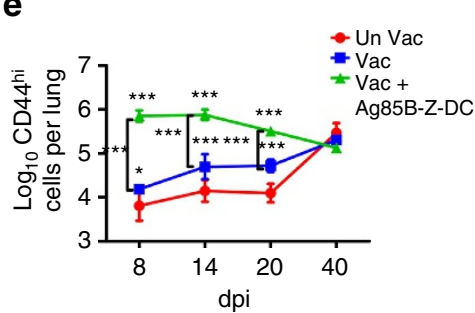

g

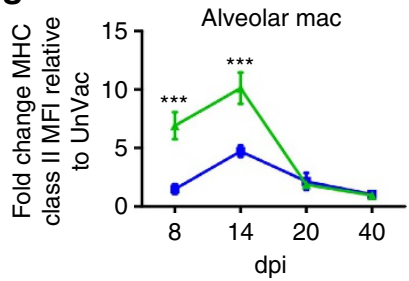

f

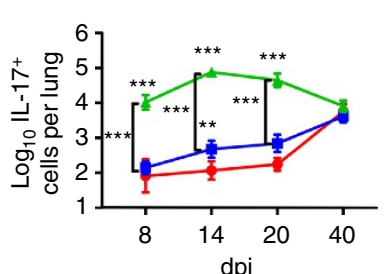

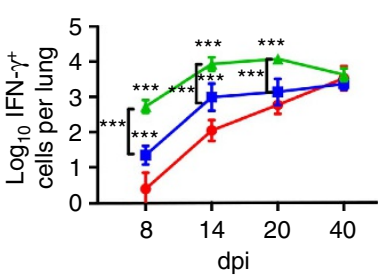

h

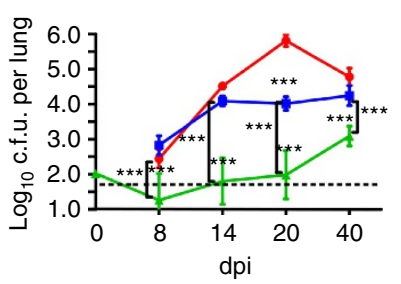

i

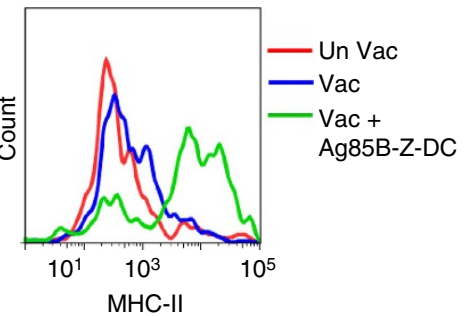

$\mathrm{Vac}+\mathrm{Ag} 85 \mathrm{~B}-\mathrm{Z}-\mathrm{DC}$
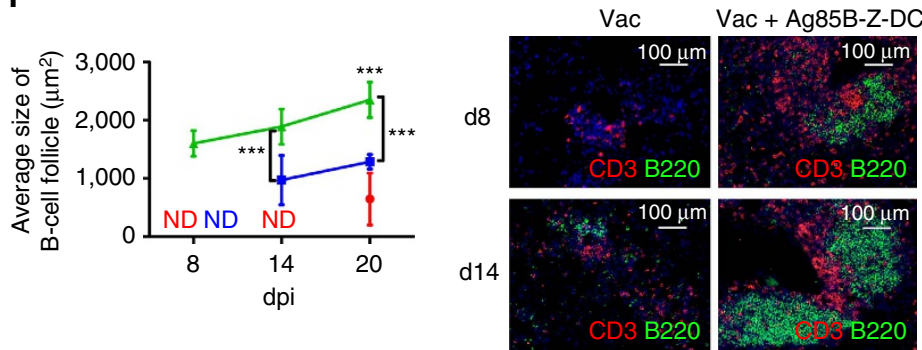

Figure 1 | Z-DC transfer in vaccinated mice confers superior Mtb control. (a-d) Naive CD4 ${ }^{+}$T cells from Ag85B TCR Tg Thy1.1+ mice were transferred into B6 mice and were vaccinated with BCG s.c. followed by mucosal boost with Ag85B240-254 peptide in mucosal adjuvant, rested for 4 weeks, before isolating total lung cells and labelling with CFSE. Cells were cultured for 3 days with either Ag85B antigen alone with or without Mtb, or BMDC pulsed with Ag85B antigen. (a) Expansion and (b) proliferation of Mtb-specific cells was assessed by flow cytometry and CFSE dilution. (c) Cytokine production was measured in supernatants. However some cytokine levels are not visible on the scale used. (d) Six day-stimulated T cells were cultured with Mtb-infected BMDMs additional 6 days and intracellular Mtb c.f.u. determined by plating. (e-i) B6 mice were vaccinated with BCG s.c. followed by mucosal boost with Ag85B 240-254 peptide in mucosal adjuvant, rested for 4 weeks and infected with $\sim 100$ c.f.u. Mtb HN878. Vaccinated mice received Ag85B-Z-DC on -1 and 4 dpi. Lungs were collected and flow cytometry was used to assess (e) CD44 expression on CD3 ${ }^{+}$CD4 ${ }^{+}$T cells, and (f) IL-17 and IFN- $\gamma$ production by Ag85B-specific CD4 ${ }^{+}$CD44 ${ }^{\text {hi }}$ T cells in the lungs. (g) Flow cytometry was used to assess MHC-II mean fluorescence intensity (MFI) on lung alveolar macrophages, and fold change relative to unvaccinated was calculated. A representative plot of MHC-II expression on alveolar macrophages from unvaccinated, vaccinated and vaccinated mice receiving Z-DC transfer is shown at $8 \mathrm{dpi}$. (h) Lung bacterial burden was determined by plating. (i) B-cell lymphoid follicle formation was determined by CD3 (red) and B220 (green) staining on formalin-fixed, paraffin-embedded sections by immunofluorescence staining. The average size of B-cell follicles per lobe was quantified using the automated tool of the Zeiss Axioplan 2 microscope in squared microns, representative images of B-cell follicles are shown. (a-d) $n=3-4$ technical replicates \pm s.d., $(\mathbf{e}-\mathbf{i}) n=4-5$ biological replicates \pm s.d. ${ }^{\star} P \leq 0.05,{ }^{\star \star} P \leq 0.01,{ }^{\star \star \star} P \leq 0.001$ by one-way analysis of variance (ANOVA) (a-d) or two-way ANOVA (e-i). ND, not detected. Dotted lines on $\mathbf{h}$ represent the limit of detection by plating.

Z-DC, induced proinflammatory cytokines such as IL-23, IL-1 $\beta$ and IL-6 (Supplementary Fig. $3 \mathrm{a}-\mathrm{c}$ ), but also induced IL-10 (Supplementary Fig. 3d). IL-10 induction can dampen vaccineinduced immunity to $M t b$ infection ${ }^{28,29}$, and we next tested whether use of B6 DCs or $I l 10^{-/-}$DCs treated with zymosan was more effective on transfer at conferring $M t b$ control in vaccinated hosts. B6 DC transfer into vaccinated $M t b$-infected mice as before reduced $M t b$ burden significantly, when compared with vaccinated $M t b$-infected hosts, while transfer of B6 Z-DCs had a small but significant improvement in $M t b$ control, when compared with vaccinated hosts receiving B6 DC transfer (Supplementary Fig. 2b). In sharp contrast, we found that 
vaccinated hosts receiving Z-DCs from $\mathrm{Il}_{10}^{-/-}$mice resulted in far superior $M t b$ control, with $M t b$ burden-bordering limit of detection by plating assays (Supplementary Fig. 2b, limit of $M t b$ detection is $\sim 75-100$ c.f.u. per lung, $M t b$ burden from three out of five mice were in this range, $\sim 99.97 \%$ reduction in $M t b$ burden, when compared with unvaccinated $M t b$-infected mice). The near-sterilizing protection in vaccinated mice receiving Illo ${ }^{-/}-$Z-DC transfer coincided with increased frequency of both activated IL-17-producing and IFN $\gamma$-producing $M t b$-specific $\mathrm{CD}^{+}{ }^{+} \mathrm{T}$ cells in the lungs (Supplementary Fig. 2c,d; gating strategy in Supplementary Fig. 4a). Indeed, B6 Z-DC transfer also resulted in increased frequency of lung IL-17-producing $M t b$-specific $\mathrm{CD}_{4}{ }^{+} \mathrm{T}$-cell responses, but failed to improve IFN $\gamma$-producing $\mathrm{CD} 4{ }^{+} \mathrm{T}$-cell responses, when compared with responses in vaccinated hosts (Supplementary Fig. 2c,d). These results together suggest that Z-DCs generated from $\mathrm{Il10}^{-/}-$mice on transfer into vaccinated hosts can overcome a TB vaccine bottleneck, and confer superior protection against $M t b$ infection by significantly accelerating the accumulation of $M t b$-specific cytokine-producing $\mathrm{CD} 4{ }^{+} \mathrm{T}$-cell vaccine responses. Thus, in all experiments in the remainder of the study, Z-DCs from $I l 10^{-/-}$ mice (Ag85B-Z-DC) were used for DC transfer.

DC transfer accelerates vaccine $\mathrm{CD} 4{ }^{+} \mathrm{T}$-cell activation. Having established the ability of Z-DC transfer to significantly reduce $M t b$ burden in the lungs of vaccinated mice, we then assessed the kinetics of immune responses in $M t b \mathrm{HN} 878$-infected vaccinated mice receiving Z-DC transfer. Vaccine-induced $\mathrm{CD}^{+}{ }^{+} \mathrm{T}$-cell responses and activation of lung myeloid cells are not detected in vaccinated hosts until $12-14$ dpi following $M t b$ challenge ${ }^{3}$. In contrast, we observed significant early accumulation (8 dpi) of activated $\mathrm{CD} 4{ }^{+} \mathrm{T}$ cells in vaccinated $M t b$-infected mice receiving Z-DC transfer, when compared with vaccinated $M t b$-infected mice (Fig. 1e; gating strategy in Supplementary Fig. 4a). In addition, increased numbers of $M t b$-specific CD44 ${ }^{\text {hi }}$-activated cells producing the cytokines IL-17 and IFN- $\gamma$ accumulated early in lungs of vaccinated $M t b$-infected mice receiving Z-DC transfer, when compared with vaccinated $M t b$-infected mice or naive $M t b$-infected mice (Fig. 1f). As noted previously, activated T-cell responses in vaccinated mice not receiving Z-DC transfer accumulated later at $14 \mathrm{dpi}$ (Fig. 1f) ${ }^{6}$, while T-cell responses between the different groups were comparable at later time points. These protective and early activation events in vaccineinduced $\mathrm{CD} 4{ }^{+}$T-cell responses coincided with increased early upregulation of major histocompatibility complex (MHC)-II expression on lung alveolar macrophages in Z-DC-recipientvaccinated $M t b$-infected mice, when compared with levels expressed in unvaccinated mice (Fig. 1g; gating strategy in Supplementary Fig. 4b). Importantly, the early and rapid activation of vaccine-induced $\mathrm{CD}^{+}$T-cell responses, and concomitant activation of alveolar macrophages was associated with complete control of $M t b$ growth, maintaining burdens at levels of initial inoculum up to $20 \mathrm{dpi}$ in vaccinated $M t b$-infected mice receiving Z-DC transfer (Fig. $1 \mathrm{~h}$ ). This is in contrast to naive and vaccinated $M t b$-infected mice, in which $M t b$ burden increased in the lungs over time (Fig. 1h). Finally, Z-DC transfer at the time of $M t b$ challenge resulted in long-lasting protection up to $40 \mathrm{dpi}$, with Z-DC transfer-recipient mice still maintaining significantly lower bacterial burdens in the lungs (Fig. 1h). As expected, at later time points, vaccinated mice showed similar $M t b$ burden as unvaccinated mice ${ }^{30}$, suggesting that while Z-DC transfer provides long-lasting vaccine-induced immunity in $M t b$ HN878-infected mice, endogenous vaccineinduced responses are not long-lasting and are lost over time. Furthermore, robust rapid formation of B-cell follicles- harbouring localized $\mathrm{T}$ cells could be detected in the lungs of vaccinated $M t b$-infected mice receiving Z-DC transfer (Fig. 1i), indicating early development of protective lung granulomas. Importantly, even on challenge with a Euro-American Mtb strain, H37Rv, we found that Z-DC transfer effectively conferred superior $M t b$ control by activating alveolar macrophages and accelerating accumulation of activated CD4 ${ }^{+}$ $\mathrm{T}$ cells (Supplementary Fig. 5a-d). Thus, our results suggest that DC transfer can effectively induce superior vaccine control on challenge with different $M t b$ strains. As $M t b$ HN878 is a clinically relevant rapidly emerging $M t b$ strain, all experiments in the remainder of the study was carried out with $M t b$ HN878. Thus, our data together suggest that DC transfer is effective in rapid restimulation of vaccine-induced $\mathrm{T}$ cells to produce cytokines and localize within lymphoid follicles to activate macrophages for rapid $M t b$ control, which is sustained long term, and across different $M t b$ strains.

DC transfer-mediated $M t b$ control is $\mathrm{CD}^{+}{ }^{+} \mathrm{T}$-cell dependent. To test the durability of Z-DC transfer in inducing superior vaccine-induced immunity against $M t b$ challenge, BCG-vaccinated mice were boosted mucosally and rested for 10 weeks after vaccination, and Z-DC transfer and Mtb HN878 infection were carried out as before. Indeed, we found that Z-DC transfer still induced superior protection in long-term-rested-vaccinated hosts, with $M t b$ burden significantly lower and at levels of detection, when compared with control vaccinated $M t b$-infected mice that were also rested for 10 weeks before $M t b$ challenge (Supplementary Fig. 6a). Importantly, vaccinated mice receiving Z-DC transfer also showed increased accumulation of activated IL- $17^{+}$and IFN- $\gamma^{+} \mathrm{CD} 4{ }^{+}$T cells, when compared with CD $4^{+}$ T-cell responses in vaccinated and unvaccinated $M t b$-infected mice (Supplementary Fig. 6b,c). These data suggest that Z-DC transfer-induced protection confers superior $M t b$ control even following a prolonged period of rest, supporting the durability of DC transfer-mediated protective efficacy in overcoming the TB vaccine bottleneck.

Previous studies have employed administration of DCs as vaccination strategy ${ }^{31,32}$, during which mice were immunized with antigen-primed DCs, and then rested before $M t b$ infection. In contrast, the approach taken here is to enhance antigen presentation at the time of $M t b$ infection by administering DCs mucosally at the time of $M t b$ infection. Thus, we tested whether use of Z-DCs as a mucosal boost to BCG vaccination, would similarly induce superior $M t b$ control. Indeed, we show that pulmonary administration of Z-DCs as a mucosal boost to BCG vaccination only had a small protective effect in vaccinated hosts, and did not match the near-sterilizing $M t b$ control seen, when Z-DC transfer was carried out at the time of $M t b$ infection in vaccinated hosts (Supplementary Fig. 6d). These data show that the improved protection conferred by Z-DC transfer is specifically due to the enhanced antigen presentation and accelerated $\mathrm{T}$ cells priming at the time of $M t b$ infection, and use of DCs as vaccination tool does not enable the superior Mtb control in vaccinated hosts.

Our data show that DC transfer into vaccinated mice at the time of $M t b$ challenge accelerates $M t b$-specific $\mathrm{CD} 4{ }^{+} \mathrm{T}$-cell responses and confers superior protection against $M t b$ challenge. Thus, we next addressed whether DC transfer-induced immunity in vaccinated hosts was dependent on vaccine-induced T-cell responses, or whether DC transfer will similarly induce nearsterilizing immunity in previously unvaccinated $M t b$-infected mice by accelerating priming of naive $\mathrm{CD}^{+}{ }^{+} \mathrm{T}$-cell responses. Thus, unvaccinated B6 mice or B6 mice that were BCG vaccinated and mucosally boosted were rested for 4 weeks, and then received Z-DC 
transfer at the time of $M t b$ challenge, and $M t b$ control was determined. Our data show that while vaccinated $M t b$-infected mice receiving Z-DC demonstrated superior bacterial control as before, $M t b$ control in unvaccinated mice receiving Z-DC transfer was less effective and resembled $M t b$ control seen in vaccinated hosts not receiving Z-DC transfer (Fig. 2a). Furthermore, although the numbers of IL-17 and IFN- $\gamma$-producing $M t b$-specific CD4 ${ }^{+}$ $\mathrm{T}$ cells in Z-DC transfer-recipient $M t b$-infected mice were significantly higher, when compared with $M t b$-infected mice not receiving $Z$-DC, the numbers were reduced, when compared with numbers seen in vaccinated mice receiving Z-DC transfer (Fig. 2b,c). In addition, T-cell localization within B-cell follicles in vaccinated mice receiving $Z$-DC transfer was higher, when compared with unvaccinated mice that received Z-DC transfer (Fig. 2d). These results together suggest that DC transfer into unvaccinated hosts can also accelerate the priming of naive $\mathrm{CD} 4^{+}$ $\mathrm{T}$ cells into cytokine-producing $M t b$-specific $\mathrm{CD} 4{ }^{+} \mathrm{T}$-cell responses and improve $M t b$ control. However, the protection in naive $M t b$-infected mice receiving Z-DC transfer is not as effective as seen in vaccinated mice receiving Z-DC transfer, suggesting that
Z-DC transfer mediating near-sterilizing immunity occurs only in previously vaccinated hosts.

To further confirm that DC transfer-mediated protection is vaccine-induced $\mathrm{CD}^{+}{ }^{+}$T-cell-dependent, vaccinated mice receiving $\mathrm{Z}$-DC transfer received a $\mathrm{CD} 4$ depleting antibody at early time points following $M t b$ challenge, and $M t b$ control in vaccinated hosts receiving Z-DC transfer was assessed at $20 \mathrm{dpi}$. Our data show that early depletion of $\mathrm{CD} 4{ }^{+} \mathrm{T}$ cells in vaccinated mice receiving DC transfer reversed the superior protection (Fig. 2e) and coincided with loss of early accumulation of IFN- $\gamma$ - and IL-17-producing $M t b$-specific CD $4^{+}$T-cell responses (Fig. 2f,g). These results together project that the near-sterilizing protection seen on DC transfer in vaccinated host is dependent on the presence of vaccine-induced $\mathrm{CD} 4{ }^{+} \mathrm{T}$-cell responses, as either absence of vaccination or absence of $\mathrm{CD} 4{ }^{+} \mathrm{T}$ cells abrogates the superior near-sterilizing protection.

DC transfer overcomes bottlenecks in different vaccinations. Our data show that Z-DC transfer accelerates vaccine-induced a

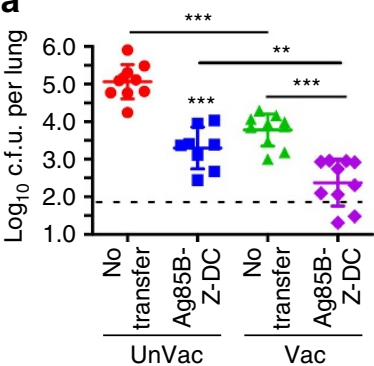

b

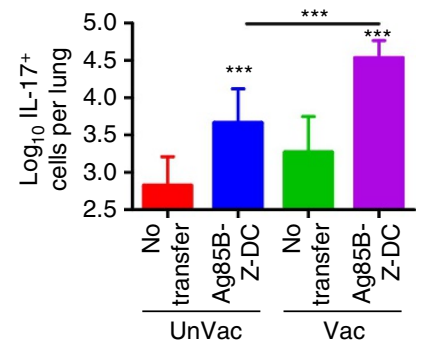

d

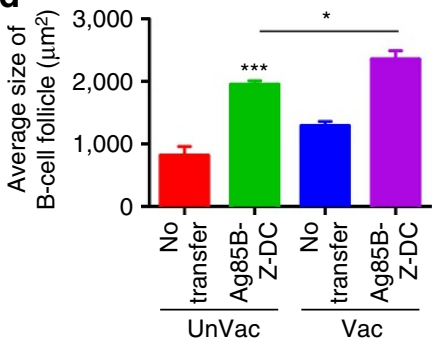

e

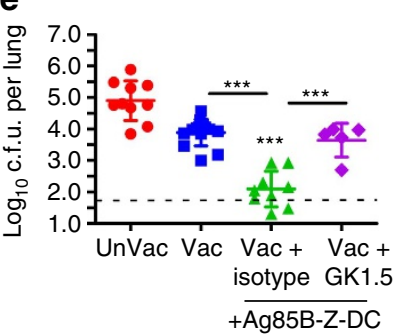

f

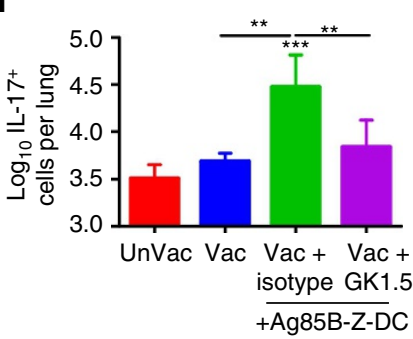

C

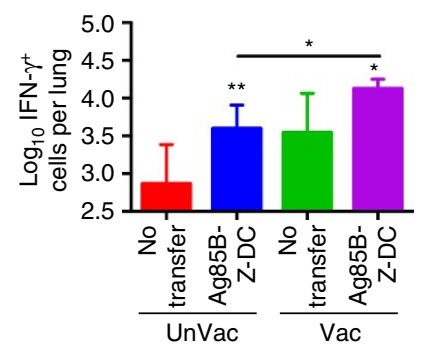

g

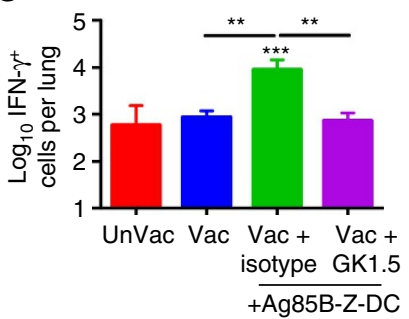

Figure 2 | Z-DC transfer-mediated superior Mtb control is CD4 ${ }^{+}$T-cell dependent. (a-d) Unvaccinated B6 mice, or B6 mice vaccinated with BCG s.C. followed by mucosal boost with $\mathrm{Ag} 85 \mathrm{~B}_{240-254}$ peptide in mucosal adjuvant, were rested for 4 weeks, and Mtb HN878-infected ( 100 c.f.u.) and treated with Ag85B-Z-DC on -1 and $4 \mathrm{dpi}$, and (a) bacterial burden was assessed by plating at $20 \mathrm{dpi}$. Flow cytometry was used to determine production of (b) IL-17 and (c) IFN- $\gamma$ by Ag85B-specific CD4 ${ }^{+}$CD44 hi T cells. (d) B-cell lymphoid follicle formation was determined by CD3 (red) and B220 (green) staining on formalin-fixed, paraffin-embedded sections by immunofluorescence staining on $20 \mathrm{dpi}$. The average size of B-cell follicles per lobe was quantified using the morphometric tool of the Zeiss Axioplan. (e-g) B6 mice were vaccinated as above, and infected with Mtb HN878 ( 100 c.f.u.) via aerosol. Mice received Ag85B-Z-DC transfer on -1 and 4 dpi; and were treated with $300 \mu \mathrm{g}$ GK1.5 (anti-CD4) or isotype control delivered i.p. on 0 and 7 dpi. Lungs were collected at $20 \mathrm{dpi}$. (e) Lung bacterial burden was determined by plating. Flow cytometry was used to assess (f) IL-17 and (g) IFN- $\gamma$ production by Ag85B-specific CD4 ${ }^{+} \mathrm{CD} 44^{\text {hi }}$ T cells. (a-d) $n=8-10$ biological replicates \pm s.d., $(\mathbf{e}-\mathbf{g}) n=5-10$ biological replicates \pm s.d., ${ }^{\star} P \leq 0.05$, ${ }^{\star \star} P \leq 0.01,{ }^{\star \star \star} P \leq 0.001$ by one-way analysis of variance or Kruskall-Wallis test (e) or Student's $t$-test (d) (UnVac + Ag85B-Z-DC compared with $\mathrm{Vac}+\mathrm{Ag} 85 \mathrm{~B}-\mathrm{Z}-\mathrm{DC}$ ). Dotted lines on bacterial burden plots represent the limit of detection by plating. 
$M t b$-specific $\mathrm{CD}^{+}{ }^{+} \mathrm{T}$-cell responses in mice vaccinated with BCG and mucosally boosted with $A g 85 B_{240-254}$ to confer superior protection on $M t b$ challenge. Thus, we next tested whether Z-DC transfer could be broadly used to provide superior protection in different vaccination strategies. Recent work by us and others has highlighted the importance of a lung-resident T-cell responses following mucosal vaccination in protection against $\mathrm{TB}$ disease ${ }^{4,5}$. In addition, mucosal vaccination induces potent lung-resident $\mathrm{CD}^{+}{ }^{+} \mathrm{T}$-cell responses, while standard parenteral BCG vaccination induces potent systemic vaccine-induced $\mathrm{CD} 4{ }^{+}$ T-cell responses (Supplementary Fig. 7a,b). Thus, we assessed whether Z-DC transfer would improve $M t b$ protection when used in standard BCG vaccination, when compared with mice that only received mucosal vaccination. $\mathrm{B} 6$ mice were vaccinated with either a mucosal $\mathrm{TB}$ vaccine consisting of $\mathrm{Ag} 85 \mathrm{~B}_{240-254}$ in mucosal adjuvant ${ }^{4}$ or parenterally with $\mathrm{BCG}^{28}$, rested, and on $M t b$ challenge received Z-DC transfer. Z-DC transfer provided superior vaccine-induced $M t b$ control in both mucosally vaccinated and BCG-vaccinated mice (Fig. 3a), suggesting that DC transfer functions broadly in different vaccination strategies. We found that while Z-DC transfer in mucosally vaccinated mice induced accumulation of large numbers of IL-17-producing $M t b-$ specific CD4 ${ }^{+}$T-cell responses, IFN- $\gamma$-producing $\mathrm{CD} 4{ }^{+}$T-cell responses were not significantly increased (Fig. 3b,c). In contrast, BCG-vaccinated mice receiving DC transfer exhibited increased both IFN- $\gamma$ and IL-17 CD4 ${ }^{+}$T-cell responses (Fig. 3b,c). Indeed, the improved $\mathrm{CD}^{+}{ }^{+} \mathrm{T}$-cell recall responses in vaccinated hosts receiving Z-DC transfer coincided with enhanced activation of alveolar macrophages when compared to unvaccinated hosts (Fig. 3d). Thus, our data show that although the effector cytokine mechanism by which Z-DC transfer confers superior protection in vaccination models may be different, Z-DC transfer can rapidly activate both systemic and mucosal $\mathrm{CD} 4{ }^{+} \mathrm{T}$-cell responses to induce superior protection against $M t b$ control in different vaccine strategies and is broadly applicable.

Identifying host pathways to overcome TB bottlenecks. Transfer of exogenously primed DCs into vaccinated mice can effectively accelerate the vaccine-induced $\mathrm{CD} 4{ }^{+} \mathrm{T}$-cell response following $M t b$ infection, and improve bacterial control. DC transfer as therapy for use in $M t b$-infected individuals in TBendemic areas; however, is logistically difficult to translate for human use as timing of $M t b$ exposure is impossible to predict. Thus, to identify endogenous host pathways that could be targeted for future use, we carried out transcriptional profiling in lungs of vaccinated $M t b$-infected mice receiving DC transfer, and compared it with gene expression profiles in lungs from $M t b$ infected-vaccinated mice. Several genes involved in early T-cell activation, macrophage function, as well as chemokines involved in B-cell follicle formation, were significantly upregulated (Supplementary Tables 1 and 2; Fig. 4a; validated in Supplementary Fig. 8). We found that the genes induced rapidly at 8 dpi in vaccinated $M t b$-infected mice receiving Z-DC transfer, were not upregulated until $21 \mathrm{dpi}$ in $M t b$-infected mice (Fig. $4 \mathrm{~b})^{33}$. These results further support our findings that protective immune responses are substantially accelerated in vaccinated $M t b$-infected mice receiving $Z$-DC transfer.

To identify pathways that were being induced in lungs of vaccinated mice receiving Z-DC transfer, we cross referenced the transcriptional signature of the vaccinated Z-DC-recipient $M t b$-infected mice (Supplementary Table 1) against all publicly available RNASeq datasets. In this unbiased search, we found the significant regulation of immune pathways associated with early T-cell activation and APC function, present in our transcriptional data also coincided with expression in the lungs of wild-type mice
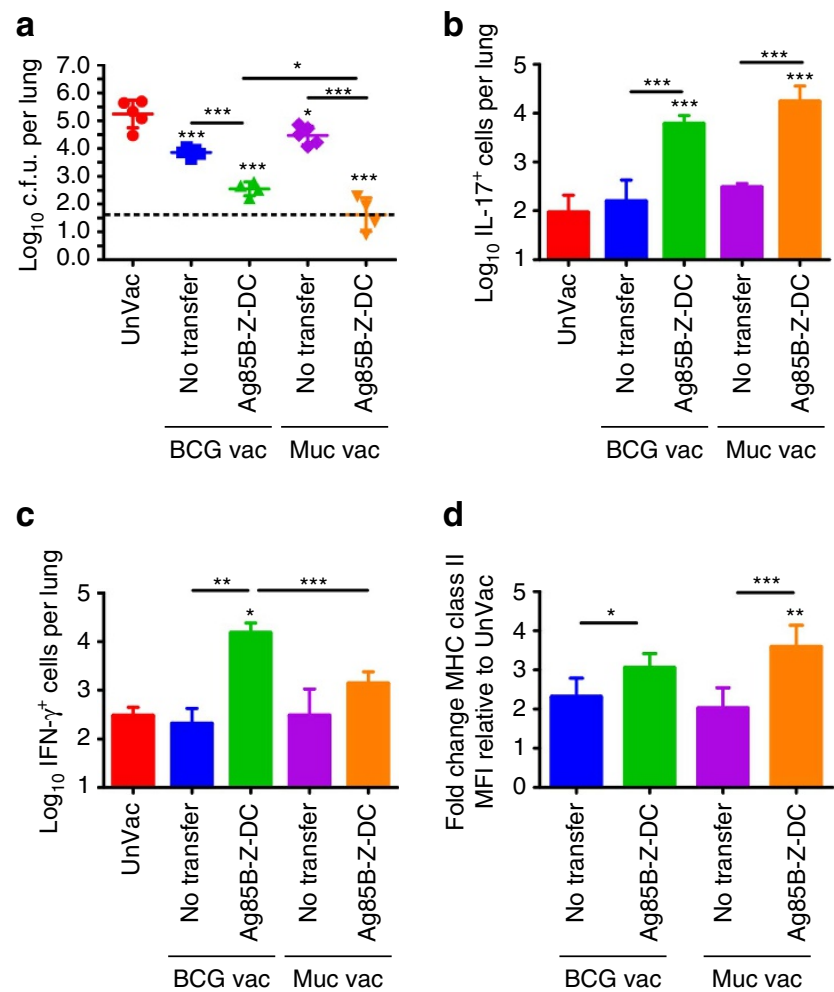

Figure 3 | Z-DC transfer improves Mtb control in different vaccines. Mice were mucosally vaccinated with three doses of $A 85 B_{240-254}$ peptide in mucosal adjuvant, or parenterally vaccinated with $B C G$ s.c., rested for 4 weeks, and were Mtb HN878-infected ( 100 c.f.u.) and received Ag85B-ZDC transfer as described previously. (a) Lung bacterial burden was assessed at 20 dpi by plating. Flow cytometry was used to determine (b) IL-17 and (c) IFN- $\gamma$ production by Ag85B-specific CD4 ${ }^{+}$CD44 ${ }^{\text {hi }}$ T cells (d) The mean fluorescence intensity (MFI) was calculated to determine level of MHC-II expression on alveolar macrophages, and fold change MFI relative to UnVac was calculated for each group at $20 \mathrm{dpi}$. $n=4-5$ biological replicates \pm s.d. ${ }^{\star} P \leq 0.05,{ }^{\star \star} P \leq 0.01,{ }^{\star \star \star} P \leq 0.001$ by one-way analysis of variance or Kruskall-Wallis test (c), or Student's $t$-test (d) (BCG Vac compared with BCG Vac + Ag85B-Z-DC). Dotted lines on bacterial burden plots represent the limit of detection by plating.

infected with the pulmonary pathogen, Pneumocystis murina. Specifically, we found that several genes were induced both in the lungs of vaccinated $M t b$-infected mice receiving Z-DC transfer, and following P. murina infection in wild-type mice. In contrast, these genes were not upregulated in $\mathrm{Cd}_{40 l^{-1-}}$ mice infected with P. murina (Fig. 4c), suggesting CD40 axis dependence underlying the accelerated vaccine response in Z-DC transferrecipient mice. Furthermore, Batf3 is a transcription factor that drives development of $\mathrm{CD}_{103}{ }^{+}$DCs, which are important for priming Th1 and Th17 responses ${ }^{34,35}$. Batf3 messenger RNA expression was increased in vaccinated $M t b$-infected hosts receiving Z-DC transfer (Supplementary Fig. 8) and coincided with increased accumulation of Batf3-dependent $\mathrm{CD} 103^{+}$DCs (Fig. 4d). Thus, we have identified a CD103 ${ }^{+} \mathrm{DC}$ and $\mathrm{CD} 40$ pathway that can potentially be targeted for improved $M t b$ control in vaccinated mice.

Activating $\mathrm{CD} 103^{+}$DCs improves $M t b$ control by vaccines. $\mathrm{CD}_{103}{ }^{+} \mathrm{DCs}$ accumulate in vaccinated $M t b$-infected hosts receiving DC transfer, and the CD40 pathway is activated in $\mathrm{Z}-\mathrm{DC}$ transfer-induced protection against $\mathrm{TB}$ disease in 
a

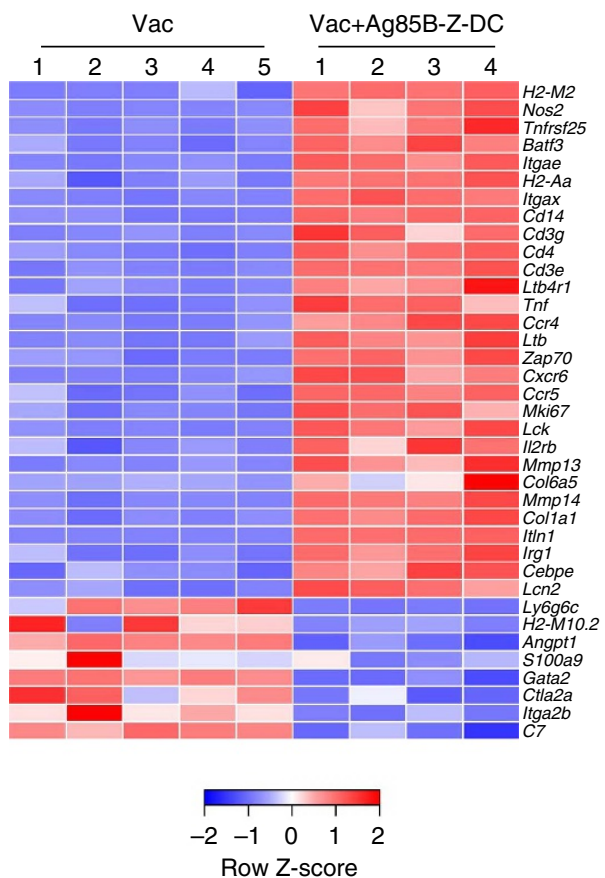

b

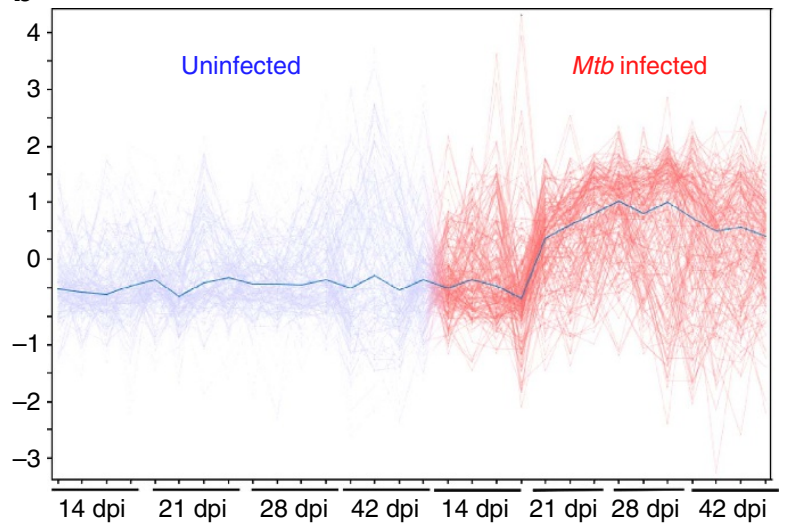

d

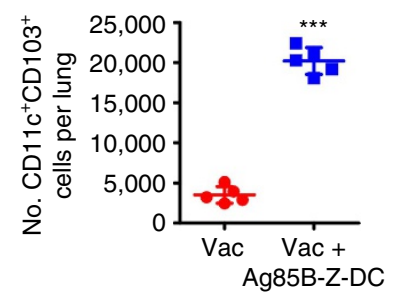

\section{C}

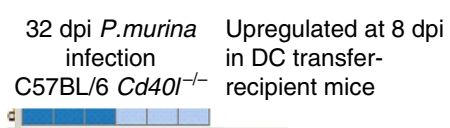

Figure 4 | Z-DC transfer induces early genes associated with APC activation. B6 mice were vaccinated with BCG s.c. followed by mucosal boost with Ag85B $240-254$ peptide in mucosal adjuvant, rested for 4 weeks, and Mtb HN878-infected ( 100 c.f.u.) and treated with Ag85B-Z-DC on -1 and 4 dpi. RNA-seq was performed on lungs at $8 \mathrm{dpi}$. (a) Expression values of each biological replicate $(n=5$ for Vac, $n=4$ for Vac + Ag85B-Z-DC) for each group of a subset of selected genes of interest (all selected genes had a differential expression of at least twofold). These genes were differentially expressed using CuffDiff output with a $P<0.05$, and Benjamini-Hochberg false discovery rate (FDR) of $5 \%$. The scaled expression of each replicate, denoted as the row $Z$-score, is plotted in red-blue colour scale with red indicating high expression and blue indicating low expression. $q$ values for each gene of interest are shown in Supplementary Table 1 for the top 100 upregulated genes. (b) Expression of the top 100 upregulated genes shown in Supplementary Table 1 was considered in the published transcriptional data following expression in lungs of mice infected (red curves) with Mtb, or uninfected controls (blue curves) along the timecourse of 14, 21, 28 and $42 \mathrm{dpi}^{33}$. (c) Gene Set Enrichment Analysis was used to analyse the expression of the top 100 upregulated genes shown in Supplementary Table 1 in the context of the infection model of $\mathrm{Cd} 4 \mathrm{Ol}^{-/-}$mice with $\mathrm{P}$. murina. Genes differentially expressed between wild type (WT) and $\mathrm{Cd}_{40 \mathrm{O}^{-/}-}$mice on 32 days post-P. murina infection were compared and the top 1,000 expressed genes shown in the heat map, ranked by the level of differential expression between the two genotypes ${ }^{70}$. Orange lines indicate positions of 100 genes from Supplementary Table $1, P<10^{-3}$. (d) Numbers of $\mathrm{CD} 11 \mathrm{c}^{+} \mathrm{CD}_{103}{ }^{+}$cells in lungs of $M t b$-infected-vaccinated Z-DC-recipient mice was determined using flow cytometry. $n=4-5$ biological replicates \pm s.d. ${ }^{\star \star \star} P \leq 0.001$ by Student's t-test. 
vaccinated hosts. To target endogenous host mucosal DCs, we then assessed if activation of the CD40 pathway and targeting endogenous $\mathrm{CD}_{103}{ }^{+}$mucosal DCs in standard BCG-vaccinated $M t b$-infected mice, can mimic the effects of DC transfer and induce superior $M t b$ control. Amphiphilic-CpG (amph-CpG) in mice is a modified Toll-like receptor (TLR) 9 agonist that is efficiently taken up by DCs and macrophages, and enhances $\mathrm{T}$-cell responses to peptide vaccines ${ }^{36}$. In addition, treatment of DCs with amph-CpG upregulates CD103 expression (Fig. 5a). FGK4.5 is an agonistic CD40 antibody that has been shown to activate DCs in murine cancer treatment models ${ }^{37,38}$. Thus, vaccinated $M t b$-infected mice received amph-CpG along with FGK4.5, or either amph-CpG or FGK4.5 alone, delivered i.t. at -1 and $4 \mathrm{dpi}$, and early vaccine responses and $M t b$ burden in the lungs were assessed. We found that while delivery of either amphCpG alone or FGK4.5 alone did not induce significant vaccine protection, delivery of amph-CpG and FGK4.5 together in vaccinated mice results in early $M t b$ control, similar to that seen in vaccinated $M t b$-infected hosts receiving DC transfer (Fig. 5b). Importantly, delivery of amph-CpG and FGK4.5 induced accelerated activation of vaccine-induced cytokineproducing $\mathrm{CD}^{+}{ }^{+} \mathrm{T}$-cell responses (Fig. 5c-e) and coincided with activation of lung alveolar macrophages (Fig. 5f). Moreover, treatment with amph-CpG and FGK4.5 resulted in increased accumulation of $\mathrm{CD}_{103}{ }^{+}$lung DCs in $M t b$-infected-vaccinated mice (Fig. 5g; see gating strategy in Supplementary Fig. 4c). Thus, our data suggest that targeting host endogenous CD103 ${ }^{+}$DCs and activation of the CD40 pathway can overcome delayed antigen presentation and results in rapid activation of vaccineinduced T-cell responses and completely control $M t b$ growth.

\section{Discussion}

Thus far, the development of sterilizing vaccines against TB for human use has not become a reality. This is especially concerning considering the status of $\mathrm{TB}$, as one of the leading causes of death due to infectious diseases in the world today. In the current study, we hypothesized that our inability to develop a sterilizing vaccine against $M t b$ infection is not due to a failure of the vaccine to induce effective $\mathrm{T}$-cell responses, but due to a delay in antigen presentation and subsequent delay in accumulation of vaccineinduced $\mathrm{T}$-cell responses. Indeed, we show here that vaccineinduced $\mathrm{T}$ cells can respond to antigen exposure and induce mycobactericidal activity ex vivo. Transfer of exogenously primed DCs into lungs of vaccinated $M t b$-infected mice can directly activate vaccine-induced $\mathrm{T}$ cells in vivo, leading to accelerated formation of protective B-cell follicles, rapid and early alveolar macrophage activation, and early control of establishment of $M t b$ infection. In addition, our results have identified novel mechanisms, involving $\mathrm{CD}_{103}{ }^{+} \mathrm{DCs}$ and the $\mathrm{CD} 40$ activation pathway that may be therapeutically targeted to improve vaccine-induced protection during TB. Together, our data provide novel and mechanistic insights into pathways that can be targeted to generate sterilizing vaccine-induced immunity against TB.

Most modern TB vaccines induce lung and systemic Th1 and/or Th17 vaccine responses ${ }^{2}$. In animal models, however, none of these vaccines induce sterilizing immunity to TB, instead only decreasing the lung $M t b$ burden by $0.5-1.0 \log$ (refs 3,8-11). Our studies show that vaccine-induced $\mathrm{CD} 4^{+} M t b$-specific $\mathrm{T}$ cells isolated from the lung can undergo activation rapidly, proliferate and produce both IFN- $\gamma$ and IL-17 ex vivo. Importantly, we show that proliferating vaccine-induced CD4 ${ }^{+}$ $\mathrm{T}$ cells can activate macrophages to control $M t b$, suggesting that there is no inherent deficiency in vaccine-induced $\mathrm{CD} 4{ }^{+}$recall responses. In addition, lung-resident vaccine-induced $\mathrm{CD}^{+}$ $\mathrm{T}$-cell responses are long lasting ${ }^{39}$ and adoptive transfer of
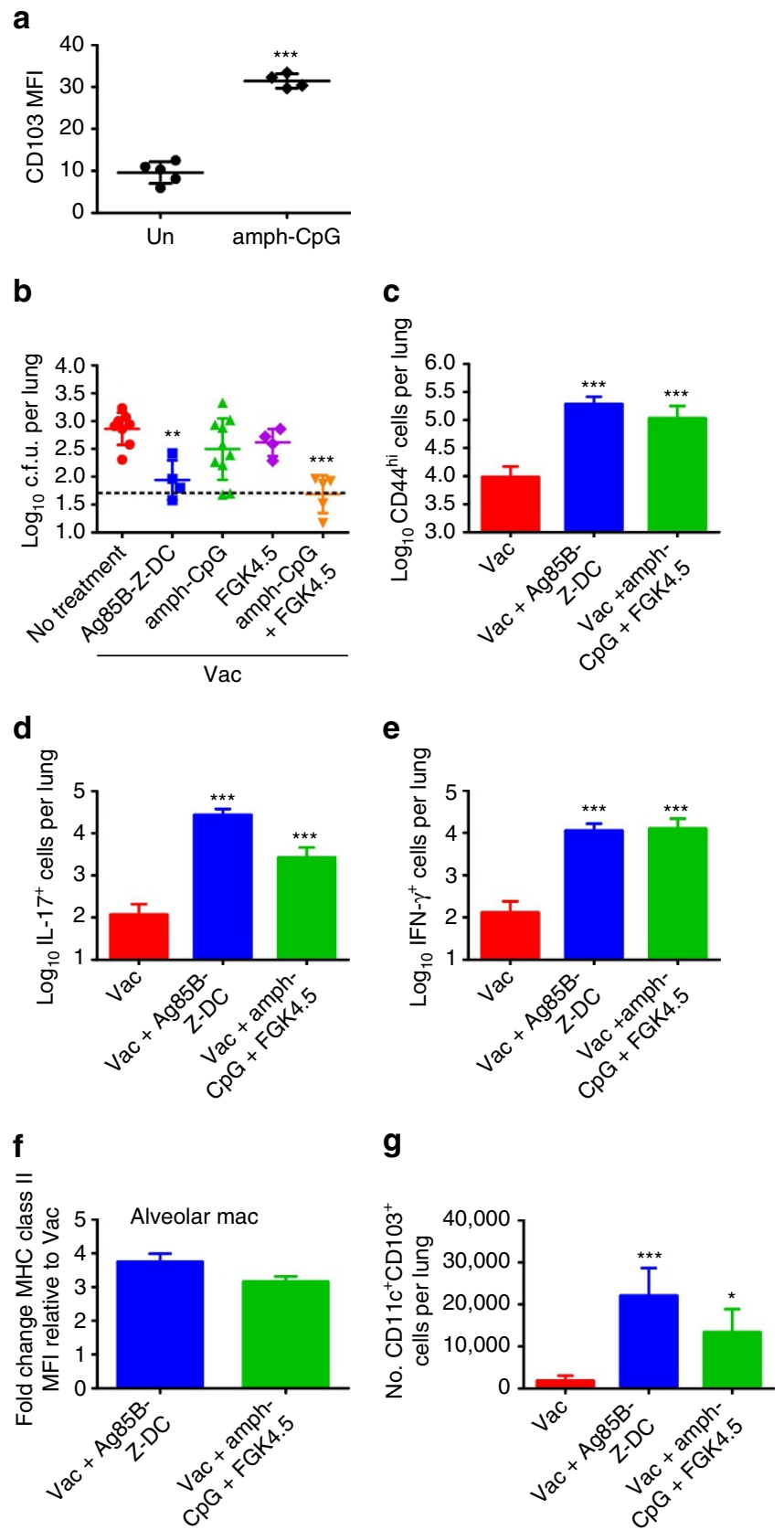

Figure 5 | Amph-CpG and CD40 agonist improves Mtb control in

vaccinated hosts. (a) BMDC were cultured from $\mathrm{B} 6$ mice. After harvesting and resting overnight, BMDC were treated overnight with $1.24 \mathrm{nmol}$ amphCpG. CD103 expression on activated DCs was assessed by flow cytometry. (b-g) Mice were vaccinated with BCG s.c. and rested for 4 weeks. Vaccinated mice were infected with Mtb HN878 and treated with either Ag85B-Z-DC, amph-CpG (1.24 nmol), FGK4.5 (100 $\mu \mathrm{g})$, or both amph-CpG and FGK4.5 along with Ag85B peptide $(5 \mu \mathrm{g})$ on -1 and $4 \mathrm{dpi}$. Mice were collected at $8 \mathrm{dpi}$. (b) Lung bacterial burden was determined by plating. Flow cytometry was used to assess (c) numbers of CD4 ${ }^{+}$CD44 ${ }^{\text {hi }}$ cells in the lungs, and (d) IL-17 and (e) IFN- $\gamma$ production by $\mathrm{CD}^{+}{ }^{+} \mathrm{T}$ cells, (f) MHC-II expression on alveolar macrophages and fold change MFI relative to Vac was calculated. (g) Flow cytometry was used to calculate numbers of $\mathrm{CD}_{11 \mathrm{c}}{ }^{+} \mathrm{CD}_{103}{ }^{+}$cells in the lungs. (a) $n=4-5$ technical replicates \pm s.d., $(\mathbf{b}-\mathbf{g}) n=4-10$ biological replicates. ${ }^{\star} P \leq 0.05,{ }^{\star \star} P \leq 0.01$, ${ }^{\star \star \star} P \leq 0.001$ by Student's $t$-test (a) or one-way analysis of variance (b-g). Dotted lines on bacterial burden plots represent the limit of detection by plating. 
T cells isolated from $M t b$-infected animals into newly infected animals confers protective immunity ${ }^{40-44}$. Mtb is a successful pathogen, known to inhibit MHC-II transactivator expression, MHC-II expression and antigen presentation in APCs ${ }^{18}$. Thus, the inability of TB vaccines to confer sterilizing immunity on $M t b$ challenge may be associated with delayed $\mathrm{CD} 4^{+} \mathrm{T}$-cell responses due to delayed APC function, rather than just poor induction of vaccine-induced $\mathrm{CD} 4^{+} \mathrm{T}$ cells. Accordingly, targeting DCs in vaccinated hosts can substantially accelerate the activation and accumulation of cytokine-producing $\mathrm{CD} 4{ }^{+}$T-cell recall responses in the lung, initiate rapid activation of alveolar macrophages and limit $M t b$ growth. Interestingly, pre-clinical vaccines that have come closest to inducing sterilizing immunity following $M t b$ infection have been recombinant mycobacterial vaccines delivered mucosally ${ }^{15,45}$. BCG is known to reduce the overall childhood morbidity caused by infections other than $M t b^{46}$, which could be due to the ability of BCG to induce innate memory' by epigenetically altering the phenotype of infected cells to one producing increased inflammatory cytokines, via autophagy and nucleotide-binding oligomerization domaincontaining protein 2 (NOD2)-dependent pathways ${ }^{47,48}$. Thus, it is possible that recombinant mycobacterial vaccines delivered mucosally target pathways similar to DC transfer through activation of lung-resident DCs and macrophages, thereby driving earlier activation of lung-resident $\mathrm{T}$ cells on $M t b$ challenge. Further work delineating the specific mechanisms by which vaccine-induced immunity can be accelerated by targeting these pathways will therefore be highly relevant.

Previous studies have attempted to improve DC function at the time of vaccination by targeting the CD40 activation pathway ${ }^{49}$, DC receptor Dec-205 (refs 50,51), or by utilizing cell-derived vaccines, such as $M t b$ antigen-primed DCs ${ }^{31,32}$. Improving DC function by targeting the CD40 pathway during vaccination had no effect ${ }^{49}$, while activating Dec-205 at the time of vaccination had a small effect $(\sim 0.5 \log$ reduction $)$ on vaccine-induced protection on $M t b$ challenge ${ }^{50,51}$. Vaccination with antigenprimed DCs has generated mixed results in $M t b$-infected mice, with a study demonstrating a negative impact of DC vaccination due to exuberant inflammation ${ }^{31}$, or induction of vaccineinduced protection similar to levels seen in BCG-vaccinated hosts $^{32}$. Our results show that use of Z-DCs as a mucosal vaccination tool as boost to BCG vaccination has a small protective effect on $M t b$ infection, but not as effective as use of Z-DC transfer at the time of infection. In contrast, only very few studies have therapeutically manipulated early innate responses following $M t b$ infection. Delivery of polyI:C, a TLR3 ligand, exacerbated inflammation and increased $M t b$ burden ${ }^{52}$. In contrast, delivery of FimH, a TLR4 ligand, improved T-cell responses and resulted in $\sim 1 \log$ reduction in lung bacterial burden, following high-dose intranasal infection with $M t b$ H37Ra (ref. 53). To our knowledge, no published studies have manipulated early host responses in vaccinated $M t b$-infected hosts, with resulting complete early $M t b$ control. Furthermore, both $\mathrm{CpG}$ (reviewed in refs 54,55) and CD40 agonists (reviewed in ref. 56) have been safely tested in clinical human trials, predominantly for cancer treatment. Thus, repurposing these host-directed therapeutics, as well as other activators of $\mathrm{CD} 103^{+}$ DC function ${ }^{57,58}$, for rapid activation of vaccine-induced T-cell responses may thus be useful in development of novel drugs or vaccination approaches for TB.

Our results show that while delivery of unstimulated DCs by itself improves vaccine-induced $\mathrm{CD} 4^{+} \mathrm{T}$-cell responses and $M t b$ control in vaccinated hosts, zymosan activation of DCs, especially generated from $\mathrm{Il} 10^{-1-}$ mice confers superior vaccine-induced $M t b$ control. These results support the idea that delayed antigen presentation and subsequent delayed induction of vaccine- induced T-cell activation is a major bottleneck for TB vaccine efficacy. In addition, these results support an important role for IL-10 in limiting TB vaccine efficacy and early $M t b$ control in vaccinated hosts ${ }^{28,29}$. Furthermore, Z-DC transfer works in both standard BCG vaccinated as well as in mucosally vaccinated $M t b$-infected hosts, the route of vaccination may define the mechanisms of protection. In mucosally vaccinated $M t b$-infected mice receiving DC transfer, mucosal lung-resident $\mathrm{CD} 4^{+} \mathrm{T}$ cells, primarily IL-17-producing cells are activated and accumulate rapidly in the lung, thus mediating the superior protection associated with this model. In BCG-vaccinated $M t b$-infected mice receiving DC transfer, DC transfer can still prime a population of lung-resident IL-17-producing vaccine-induced $\mathrm{CD} 44^{+} \mathrm{T}$ cells, but likely DC migration to LNs is required to mobilize IFN- $\gamma$ producing vaccine-induced $\mathrm{CD} 4{ }^{+}$T-cell responses from the systemic pool, to provide enhanced $M t b$ control. Regardless, that we can target $\mathrm{CD} 103^{+}$DCs and the $\mathrm{CD} 40$ activation pathway to limit $M t b$ growth in standard BCG-vaccinated $M t b$-infected hosts suggest that our strategy is effective in overcoming a bottleneck associated with delayed induction of vaccine-induced $\mathrm{CD} 4{ }^{+}$ T-cell responses in vaccinated hosts. This is further supported by our results showing that DC transfer can protect vaccinated hosts on challenge with $M t b$ strains belonging to different lineages, suggesting that T-cell bottleneck for TB vaccines is universal. In addition, that Z-DC transfer into unvaccinated hosts can also accelerate naive T-cell priming and improve primary immunity, albeit not to the effectiveness seen in vaccinated hosts, suggest the DC transfer may be useful to enhance both primary and vaccineinduced pathways during $M t b$ infection.

DC transfer not only induces the rapid activation of vaccineinduced $\mathrm{CD} 4{ }^{+} \mathrm{T}$-cell responses in vaccinated $M t b$-infected mice, but also has substantial downstream effects on activation of myeloid pathways in the lung. First, lung alveolar macrophages are rapidly activated in vaccinated hosts by $8 \mathrm{dpi}$, an event that does not happen until $14 \mathrm{dpi}$ in vaccinated hosts and until $21 \mathrm{dpi}$ in unvaccinated hosts ${ }^{3}$. Accordingly, the early global transcriptional changes in the lungs of vaccinated $M t b$-infected mice receiving DC transfer mirror the transcriptional changes occurring in $21 \mathrm{dpi} M t b$-infected lungs ${ }^{33}$. Furthermore, we observe early accumulation of myeloid DCs, including $\mathrm{CD}_{103}{ }^{+}$ DCs and an enhancement of DC activation pathways, in particular genes associated with CD40 activation in the lungs of vaccinated $M t b$-infected mice. $\mathrm{CD} 103^{+}$DCs are a tissue-resident DC subset, which have primarily been implicated in crosspresentation and induction of $\mathrm{CD} 8^{+} \mathrm{T}$-cell responses ${ }^{59,60}$. More recently, however, $\mathrm{CD}_{103}{ }^{+} \mathrm{DCs}$ have been shown to induce $\mathrm{CD}^{+}{ }^{+} \mathrm{T}$ cells $s^{34,35,61}$. In the lung, CD103 ${ }^{+}$DCs represent a migratory DC subset, with the ability to migrate from the lungs to the LN and back again ${ }^{62}$, and induce both Th1 and Th17 cytokines by $\mathrm{CD} 44^{+} \mathrm{T}$ cells $s^{34,35,63}$. Although antigen presentation by transferred DCs likely directly activates vaccine-induced $\mathrm{CD} 4^{+} \mathrm{T}$-cell responses, rapid activation of $\mathrm{CD} 4^{+} \mathrm{T}$-cell responses also modifies the lung micro-environment to improve subsequent antigen presentation by endogenous host APCs. We show here that targeting DC activation pathways through the TLR ligand amph-CpG, along with use of the CD40 agonist FGK4.5, similarly control $M t b$ growth. Treatment of DCs with granulocyte-macrophage-colony-stimulating factor (GM-CSF), a by-product of TLR9 stimulation through $\mathrm{CpG}^{64}$ has previously been shown to upregulate CD103 expression ${ }^{65}$. Thus, it is possible that $\mathrm{CpG}$ stimulates $\mathrm{CD}_{103}{ }^{+}$expression on DCs indirectly through inducing GM-CSF by other lung-resident cells. Upregulation of $\mathrm{CD} 103$ as well as recruitment of $\mathrm{CD} 103^{+}$ DCs to the lung alone, however, are not sufficient for improved T-cell activation, suggesting that the activating signal delivered through the CD40 agonist FGK4.5 provides the second signal 
required for improved early antigen presentation to vaccineinduced $\mathrm{CD} 4{ }^{+} \mathrm{T}$ cells. Therefore, $M t b$-infected individuals likely have a population of antigen-responsive $\mathrm{T}$ cells, and targeting DCs as a potential therapy, may provide the lung-resident $T$ cells the necessary signals to proliferate and control $M t b$ infection.

Thus, our data for the first time show that delayed activation of vaccine-induced $\mathrm{T}$-cell responses is a critical bottleneck for TB vaccine efficacy. Our results demonstrate that this delay can be overcome by DC transfer, or by targeting DC activation, leading to rapid $\mathrm{T}$-cell recall responses and enhanced alveolar macrophage activation, resulting in complete control of early $M t b$ growth. These results provide a roadmap for the type of immune responses that a sterilizing $\mathrm{TB}$ vaccine should induce, representing an important milestone in our mechanistic understanding of TB vaccine-mediated immune responses. Our results suggest that despite induction of effective $M t b$-specific vaccine T-cell responses by modern $\mathrm{TB}$ vaccines ${ }^{66-68}$, delayed $\mathrm{T}$-cell vaccine responses on $M t b$ infection may pose a significant hurdle to development of a sterilizing vaccine for TB in humans. Therefore, it is possible that the development of a sterilizing vaccine for TB may not be a realistic goal. Instead, redirecting our efforts towards developing novel vaccine strategies and therapeutics for preventing the reactivation of latent $\mathrm{TB}$ and $\mathrm{TB}$ transmission may benefit global control of TB.

\section{Methods}

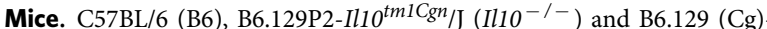
Foxp3 $3^{\text {tm } 3(D T R / G F P) A y r} / \mathrm{J}$ (Foxp3.DTR) mice were purchased from Jackson Laboratories (Bar Harbor, ME, USA) and bred at Washington University in St Louis. C57BL/6-Tg(H2-Kb-Tcra,-Tcrb)P25Ktk/J (P25 TCR Tg) and B6.PLThy $1^{a} /$ CyJ (Thyl.1) mice were purchased from Jackson Laboratories, and crossed and bred at Washington University in St Louis. Mice (male and female) were used at 6-8 weeks of age. Experiments were designed according to empirical statistical power analysis. All mice were used and housed in accordance with the National Institute of Health guidelines for housing and care of laboratory animals, and permission for the experiments in this study was granted by the Washington University in St Louis Institutional Animal Care and Use Committee under protocol 20130195. All efforts were made to minimize pain and suffering as described in this protocol.

Bacterial infection and vaccination. M. bovis Bacille Calmette-Guerin (BCG Pasteur, Source: Trudeau institute), $M t b$ strain HN878 (Source: BEI Resources) and $M t b$ strain H37Rv (Source: Trudeau Institute) were grown to mid-log phase in Proskauer Beck medium containing $0.05 \%$ Tween 80 and frozen in $1 \mathrm{ml}$ aliquots at $-80^{\circ} \mathrm{C}$.

Mice were vaccinated with $1 \times 10^{6}$ c.f.u. BCG s.c. ${ }^{4}$ and 4 weeks later received $133 \mu \mathrm{g}$ Ag85B $240-254$ peptide (New England Peptide, Gardner, MA, USA) along with $1 \mu \mathrm{g}$ heat-labile enterotoxin (LT-IIb) intranasally in $20 \mu \mathrm{l}(10 \mu \mathrm{l}$ per nare). In some experiments, mice only received mucosal vaccination with three doses, 2 weeks apart of $133 \mu \mathrm{g} \mathrm{Ag} 8 \mathrm{~B}_{240-254}$ peptide in $1 \mu \mathrm{g}$ LT-IIb intranasally. In other experiments, mice only received $1 \times 10^{6}$ c.f.u. BCG s.c. as a model of parenteral vaccination. Four or 10 weeks after the final vaccination, mice were infected with 100 c.f.u. (low dose) or 1,000 c.f.u. (high dose) Mtb HN878 via aerosol using a Glas-Col aerosol exposure system (Glas-Col, Terre Haute, IN, USA) ${ }^{4}$. At given time points following infection, organs were collected, homogenized and tissue homogenates plated in serial dilutions on 7H11 agar (BD Biosciences, San Jose, CA, USA) to assess bacterial burden ${ }^{4}$.

In experiments using Foxp3.DTR mice, mice were treated with three doses 2 days apart of $10 \mu \mathrm{g} \mathrm{kg}^{-1}$ diphtheria toxin (Millipore, Billerica, MA, USA) ${ }^{69}$ for depletion of Foxp3 ${ }^{+}$cells, starting 8 dpi.

In vitro culture of DCs and macrophages. BMDC and bone marrow-derived macrophages (BMDMs) were generated from $\mathrm{B} 6$ or $1 l 10^{-/-}$mice. Cells were isolated from the femur and tibia, and cultured at $1 \times 10^{6}$ cells ml ${ }^{-1}$ in $10 \mathrm{ml}$ complete DMEM (cDMEM) supplemented with $20 \mathrm{ng} \mathrm{ml}^{-1}$ recombinant mouse GM-CSF (Peprotech, Rocky Hill, NJ, USA) for 3 days at $37^{\circ} \mathrm{C}$ in $7.5 \% \mathrm{CO}_{2}$, at which point, an additional $10 \mathrm{ml}$ cDMEM supplemented with $20 \mathrm{ng} \mathrm{ml}^{-1}$ recombinant mouse GM-CSF was added. Non-adherent cells (BMDCs) were collected on the seventh day of culture, and counted and plated at $2 \times 10^{6}$ cells $\mathrm{ml}^{-1}$ in cDMEM. Adherent BMDMs were collected at the same time by scraping and cultured at $1 \times 10^{6}$ cells ml $^{-1}$. For BMDC stimulation, Ag85B $\mathrm{B}_{240-254}$ peptide $\left(20 \mu \mathrm{g} \mathrm{ml}^{-1}\right)$ and Zymosan (Invivogen, San Diego, CA) was added at $25 \mu \mathrm{g} \mathrm{ml}^{-1}$. Cells were stimulated for $16 \mathrm{~h}$ before being collected, washed, counted and instilled i.t. into mice at $1 \times 10^{6}$ cells in $50 \mu \mathrm{l}$ on the day before infection and 4 dpi. Supernatants from stimulated in vitro cultures were collected and frozen at $-80^{\circ} \mathrm{C}$ for analysis by enzyme-linked immunosorbent assay and multiplex assay. In some experiments, BMDCs were treated with $1.24 \mathrm{nmol}$ amph-CpG.

Amph-CpG and FGK4.5 treatment of mice. Amph-CpG was prepared as described previously ${ }^{36}$. Briefly, solid phase DNA synthesis and $5^{\prime}$ lipophilic conjugation were carried out using an ABI 394 synthesizer $^{36}$. The sequence used was murine oligodeoxynucleotides (ODN) class $B$ sequence 1,826 with two guanine spacers: $5^{\prime}$-diacyl lipid- ${ }^{\star} G^{\star} G^{\star} T^{\star} C^{\star} C^{\star} A^{\star} T^{\star} G^{\star} A^{\star} C^{\star} G^{*} T^{\star} T^{\star} C^{\star} C^{\star} T^{\star} G^{*} A^{\star} C^{\star} G^{\star} T^{\star}$ T- $3^{\prime}$. Amph-CpG was delivered to mice in $50 \mu \mathrm{l}$ i.t. at $1.24 \mathrm{nmol}$ per mouse. The CD40 agonist FGK4.5 (R\&D Systems, Minneapolis, MN, USA) was delivered in $50 \mu \mathrm{l}$ i.t. at $100 \mu \mathrm{g}$ per mouse. Both treatments were delivered with $5 \mu \mathrm{g}$ Ag85B peptide.

Antibody treatment of mice. In some experiments, mice were treated with CD4 (clone GK1.5, BioXcell) depleting antibodies delivered intraperitoneally, starting on the day of $M t b$ infection and 7 days later $(300 \mu$ g per mouse).

Generation of single-cell suspensions from tissues. Lung single-cell suspensions from vaccinated or $M t b$-infected mice were generated as before ${ }^{4}$. For LNs and spleens, organs were passed through a $70 \mu \mathrm{m}$ cell strainer and then processed as for lungs ${ }^{4}$. For flow cytometric analysis, cells were either stained immediately, or stimulated for $18 \mathrm{~h}$ in the presence of $\mathrm{Ag} 85 \mathrm{~B}_{240-254}$ peptide, with GolgiStop $\left(5 \mu \mathrm{ml}^{-1}\right.$; BD Biosciences) and Brefeldin A (1 $\mu \mathrm{g} \mathrm{ml}^{-1}$; BioLegend, San Diego, $\mathrm{CA}$, USA) added for the last $5 \mathrm{~h}$. Intracellular cytokine staining was performed using the BD Cytofix/Cytoperm kit (BD Biosciences). Cells were collected using a BD FACS Jazz or a BD LSR Fortessa, and analysis was performed using FlowJo (Treestar, Ashland, OR, USA). Antibodies used include anti-mouse CD11c (clone HL3; BD Biosciences; dilution: 1/200), anti-mouse MHC-II (clone M5/114.15.2; Tonbo Biosciences, San Diego, CA, USA; dilution: 1/150), anti-mouse CD3 (clone 500A2; BD Biosciences; dilution: 1/200), anti-mouse CD4 (clone RM4-5; BD Biosciences, dilution: 1/200), anti-mouse CD44 (clone IM7; eBioscience, dilution: 1/400), anti-mouse IL-17 (clone TC11-18H10; BD Biosciences, dilution: 1/100), anti-mouse IFN- $\gamma$ (XMG1.2; BD Biosciences; dilution: 1/100), anti-mouse Thyl.1 (clone OX-7; BD Biosciences; dilution: 1/100) and anti-mouse CD103 (clone 2E7, eBioscience; dilution: 1/200).

ELISpot. For analysis of production of antigen-specific cytokines by enzymelinked immunospot (ELISpot) assay as before ${ }^{4}$, 96-well ELISpot plates were coated with monoclonal anti-mouse IL-17 antibody (clone 50101.111; R\&D systems, Minneapolis, MN, USA) or anti-mouse IFN- $\gamma$ antibody (clone R4-6A2; eBioscience) overnight, then blocked with media containing $10 \%$ fetal bovine serum. Total cells from lungs, or $5 \times 10^{5}$ cells from LN were plated in the top well and subsequently diluted twofold in serial dilutions. Irradiated B6 splenocytes were used as APCs at a concentration of $1 \times 10^{6}$ cells per well in the presence of $10 \mu \mathrm{g} \mathrm{ml}^{-1}$ peptide and $10 \mathrm{U} \mathrm{ml}^{-1} \mathrm{IL}-2$. After $24 \mathrm{~h}$, plates were washed and probed with biotinylated anti-mouse IL-17 antibody (clone eBio17B7; eBioscience) or biotinylated anti-mouse IFN- $\gamma$ antibody (clone XMG1.2; eBioscience), and binding detected using streptavidin-alkaline phosphatase (Vector Laboratories, Burlingame, CA, USA) with BCIP/NBT (Sigma Aldrich) as the substrate. Spots were visualized and enumerated using a CTL-Immunospot S5 MicroAnalyzer (C.T.L., Shaker Heights, OH, USA).

Cell proliferation and mycobacterial killing assays. CD4 ${ }^{+} \mathrm{T}$ cells were isolated from single-cell suspensions generated from LNs and spleen from Ag85B TCR Tg mice on a Thyl.1 background using a CD4 L3T4 positive selection kit according to the manufacturer's instructions (Miltenyi Biotec Inc, San Diego, CA, USA). Naive $\mathrm{CD}^{+} \mathrm{T}$ cells $(\mathrm{Th} 0)$ were intravenously transferred into B6 mice (on a Thy1.2 background) at $2 \times 10^{6}$ cells per mouse. For assessment of proliferation ex vivo, whole lung cells isolated from mice receiving adoptively transferred $\mathrm{T}$ cells were labelled with $1 \mu \mathrm{M}$ carboxyfluorescein succyinimidyl ester (CFSE, Invitrogen,

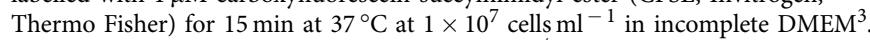
Cells were then cultured for 3 or 6 days with $I l 10^{-/-}$BMDC stimulated overnight with zymosan alone $\left(25 \mu \mathrm{g} \mathrm{ml}^{-1}\right)$ or zymosan $\left(25 \mu \mathrm{g} \mathrm{ml}^{-1}\right)+\mathrm{Ag}_{85 B_{240-254}}$ peptide $\left(20 \mu \mathrm{g} \mathrm{ml}^{-1}\right)$ in the presence or absence of $M t b \mathrm{HN} 878$. Supernatants were collected and frozen at $-80^{\circ} \mathrm{C}$. Cells were collected and T-cell proliferation was assessed by measuring carboxyfluorescein succyinimidyl ester dilution by flow cytometry. Transferred antigen-specific cells were tracked in vivo using Thyl.1 staining. Cell activation was measured by CD44 staining.

For the mycobacterial killing assay, BMDMs were infected overnight with $M t b$ HN878 at an multiplicity of infection of 1 in antibiotic-free cDMEM. The following day, infected BMDMs were co-cultured for 6 days at a 1:1 ratio with T cells previously activated with Ag85B-Z-DC as described above. Intracellular Mtb c.f.u. was assessed by lysing the BMDMs with $0.05 \%$ SDS before plating on $7 \mathrm{H} 11$ plates in serial dilutions.

Cytokine and chemokine production. Cytokine and chemokine production in supernatants collected from stimulated BMDC or from T-cell-BMDC cultures was 
analysed using Milliplex Multiplex Assays (Millipore), using the protocol specified by the manufacturer. IL-10 (R\&D; cat. no: DY417), IL-23 (R\&D; cat. no: DY1887) and IL-6 (R\&D; cat. no: DY406) were measured using a Duoset kit (R\&D Systems), using the protocol specified by the manufacturer. IL-1 $\beta$ (BD; cat. no: 559603) was measured using an BD OptEIA IL-1 $\beta$ ELISA Set (BD Biosciences) using the protocol specified by the manufacturer.

Immunofluorescence staining. Lung lobes were perfused with $10 \%$ formalin, fixed and paraffin embedded. For immunofluorescent staining, lung sections were cut, immersed in xylene to remove paraffin and then hydrated in $96 \%$ alcohol and phosphate-buffered saline. Antigens were unmasked with a DakoCytomation Target Retrieval Solution (Dako, Carpinteria, CA, USA), and non-specific binding was blocked with $5 \%(\mathrm{v} / \mathrm{v})$ normal donkey serum and Fc block (BD Pharmingen). Endogenous biotin was neutralized by adding avidin, followed by incubation with biotin (Sigma Aldrich). Sections were probed with anti-B220 to detect B cells (clone RA3-6B2, BD Pharmingen; dilution: 1/100) and anti-CD3 to detect T cells (clone M-20, Santa Cruz Biotechnology, Santa Cruz, CA; dilution: 1/100). For analysis of B-cell follicles, follicles were outlined with the automated tool of the Zeiss Axioplan 2 microscope (Zeiss, Thornwood, NY, USA), and total area and average size was calculated in squared microns.

Quantitative PCR with reverse transcription. Total RNA was isolated from lung tissue using an RNeasy RNA isolation kit (Qiagen, Valencia, CA, USA). Complementary DNA was generated using $\mathrm{ABI}$ reverse transcription reagents (ABI, Thermo Fisher) on a BioRad DNA Engine Thermal Cycler (BioRad, Hercules, CA, USA). Gene expression was assessed using primers from integrated DNA technologies (IDT) (iNOS (F-CAGCTGGGCTGTACAAACCTTC; R-CATTGGAAGTGAAGCGTTTCG;PROBE5'-/56-FAM/CGG GCA GCC TGT GAG ACC TTT GA/3BHQ_1/-3');GAPDH (F-CTCGTCCCGTAGACAAAATGG; R-AATCTCCACTTTGCCACTGCA; PROBE5' -/56-FAM/CGG ATT TGG CCG TAT TGG GCG/3BHQ 1/-3')) (Coralville, IA, USA) and ABI (CCL19: Mm00839967_g1; Saa3: Mm00441203-ml; Lcn2: Mm01324472_g1;Batf3: Mm01318274_m1; Itgae: Mm00434443_m1; Colla1: Mm00801666_g1) and run on a Viia7 Real-Time PCR system (Life Technologies, Thermo Fisher). Expression of genes of interest was normalized to GAPDH expression, and log 10-fold induction over the control group was assessed using the $\Delta \Delta \mathrm{CT}$ calculation.

RNA-seq and gene set enrichment analysis. Total RNA was isolated from lung tissue using an RNeasy RNA isolation kit (Qiagen, Valencia, CA, USA). Each sample was assessed using Qubit 2.0 fluorometer (Invitrogen, Thermo Fisher) and Agilent Tapestation 2200 (Agilent Technologies, Santa Clara, CA, USA). Sequencing libraries were generated using Illumina TruSeq RNA Access library prep kit (Illumina, San Diego, CA, USA) following the manufacturer's protocol. Cluster generation and $75 \mathrm{bp}$ single read single-indexed sequencing was performed on Illumina NextSeq 500 (Illumina). Sequencing analysis was done using mRNA-seq Analysis on Maverix Analytic Platform (Maverix Biomics, Inc, San Mateo, CA). Raw sequencing reads from Illumina sequencing platform that was converted into FASTQ file format were quality checked for potential sequencing issues and contaminants using FastQC. Adapter sequences, primers, Ns and reads with quality score $<28$ were trimmed using fastq-mcf of ea-utils and Trimmomatic. Reads with a remaining length of fewer than $20 \mathrm{bp}$ after trimming were discarded. Single reads were mapped to the mouse genome (m10) using STAR in a strand specific manner. Cufflinks was used to determine fragments per kilobase of transcript per million mapped reads (FPKM) levels for each gene from the STAR alignment and was used as input for Cuffdiff. Pairwise differential expression was quantified using Cuffdiff. Read counts were then normalized across all samples and significant differentially expressed genes were determined by adjusted $P$ value with a threshold of 0.05 . For Gene Set Enrichment Analysis we have selected expressed genes from GSE11005 (top 10,000 based on average expression level). We then assembled ranked list using signed statistics values and performed pre-ranked GSEA using top 100 vaccine upregulated genes as a query gene set. The data shown is uncorrected for multiple comparisons. We also performed differential expression analysis using Limma between wild type and $\mathrm{Cd}_{40 l^{-/}}$groups at day 32 post-

P. murina infection.

Statistical analysis. Statistical analyses were performed using GraphPad Prism (La Jolla, CA, USA). For experiments with two groups, two-tailed Student's $t$-tests or Mann-Whitney test were performed. For two or more groups, a one-way analysis of variance or Kruskall-Wallis test was used. For multiple groups with multiple time points, a two-way analysis of variance was used. All plots show mean \pm s.d.

Data availability. Sequence data that support the findings of this study have been deposited in BioProject with the primary accession code SRP091809. Other data that support the findings of this study are available from the corresponding author upon request.

\section{References}

1. Dye, C. \& Williams, B. G. The population dynamics and control of tuberculosis. Science 328, 856-861 (2010).

2. Griffiths, K. L. \& Khader, S. A. Novel vaccine approaches for protection against intracellular pathogens. Curr. Opin. Immunol. 28, 58-63 (2014).

3. Khader, S. A. et al. IL-23 and IL-17 in the establishment of protective pulmonary $\mathrm{CD}_{4}{ }^{+} \mathrm{T}$ cell responses after vaccination and during Mycobacterium tuberculosis challenge. Nat. Immunol. 8, 369-377 (2007).

4. Gopal, R. et al. Interleukin-17-dependent CXCL13 mediates mucosal vaccineinduced immunity against tuberculosis. Mucosal Immunol. 6, 972-984 (2013).

5. Aguilo, N. et al. Pulmonary but Not Subcutaneous Delivery of BCG Vaccine Confers Protection to Tuberculosis-Susceptible Mice by an Interleukin 17Dependent Mechanism. J. Infect. Dis. 213, 831-839 (2015).

6. Monin, L. et al. Immune requirements for protective Th17 recall responses to Mycobacterium tuberculosis challenge. Mucosal Immunol. 8, 1099-1109 (2015).

7. Desel, C. et al. Recombinant BCG DeltaureC hly + induces superior protection over parental BCG by stimulating a balanced combination of type 1 and type 17 cytokine responses. J. Infect. Dis. 204, 1573-1584 (2011).

8. Aagaard, C. et al. A multistage tuberculosis vaccine that confers efficient protection before and after exposure. Nat. Med. 17, 189-194 (2011).

9. Bertholet, S. et al. A defined tuberculosis vaccine candidate boosts BCG and protects against multidrug-resistant Mycobacterium tuberculosis. Sci. Transl. Med. 2, 53ra74 (2010)

10. Goonetilleke, N. P. et al. Enhanced immunogenicity and protective efficacy against Mycobacterium tuberculosis of bacille Calmette-Guerin vaccine using mucosal administration and boosting with a recombinant modified vaccinia virus Ankara. J. Immunol. 171, 1602-1609 (2003).

11. Tchilian, E. Z. et al. Immunogenicity and protective efficacy of prime-boost regimens with recombinant (delta)ureC hly + Mycobacterium bovis BCG and modified vaccinia virus ankara expressing $M$. tuberculosis antigen $85 \mathrm{~A}$ against murine tuberculosis. Infect. Immun. 77, 622-631 (2009).

12. Sweeney, K. A. et al. A recombinant Mycobacterium smegmatis induces potent bactericidal immunity against Mycobacterium tuberculosis. Nat. Med. 17, 12611268 (2011).

13. Grode, L. et al. Increased vaccine efficacy against tuberculosis of recombinant Mycobacterium bovis bacille Calmette-Guerin mutants that secrete listeriolysin. J. Clin. Invest. 115, 2472-2479 (2005).

14. Martin, C. et al. The live Mycobacterium tuberculosis phoP mutant strain is more attenuated than BCG and confers protective immunity against tuberculosis in mice and guinea pigs. Vaccine 24, 3408-3419 (2006).

15. Kaushal, D. et al. Mucosal vaccination with attenuated Mycobacterium tuberculosis induces strong central memory responses and protects against tuberculosis. Nat. Commun. 6, 8533 (2015).

16. Reiley, W. W. et al. ESAT-6-specific CD4 T cell responses to aerosol Mycobacterium tuberculosis infection are initiated in the mediastinal lymph nodes. Proc. Natl Acad. Sci. USA 105, 10961-10966 (2008).

17. Wolf, A. J. et al. Initiation of the adaptive immune response to Mycobacterium tuberculosis depends on antigen production in the local lymph node, not the lungs. J. Exp. Med. 205, 105-115 (2008).

18. Harding, C. V. \& Boom, W. H. Regulation of antigen presentation by Mycobacterium tuberculosis: a role for Toll-like receptors. Nat. Rev. Microbiol. 8, 296-307 (2010).

19. Urdahl, K. B. Understanding and overcoming the barriers to T cell-mediated immunity against tuberculosis. Semin. Immunol. 26, 578-587 (2014).

20. Srivastava, S., Grace, P. S. \& Ernst, J. D. Antigen export reduces antigen presentation and limits T cell control of M. tuberculosis. Cell Host Microbe 19, 44-54 (2016)

21. Jung, Y. J., Ryan, L., LaCourse, R. \& North, R. J. Properties and protective value of the secondary versus primary $\mathrm{T}$ helper type 1 response to airborne Mycobacterium tuberculosis infection in mice. J. Exp. Med. 201, 1915-1924 (2005).

22. Srivastava, S. \& Ernst, J. D. Cell-to-cell transfer of $M$. tuberculosis antigens optimizes CD4 T cell priming. Cell Host Microbe 15, 741-752 (2014).

23. Vilaplana, C. et al. To achieve an earlier IFN-gamma response is not sufficient to control Mycobacterium tuberculosis infection in mice. PLoS ONE 9, e100830 (2014).

24. Shafiani, S., Tucker-Heard, G., Kariyone, A., Takatsu, K. \& Urdahl, K. B. Pathogen-specific regulatory $\mathrm{T}$ cells delay the arrival of effector $\mathrm{T}$ cells in the lung during early tuberculosis. J. Exp. Med. 207, 1409-1420 (2010)

25. Quinn, K. M. et al. Inactivation of $\mathrm{CD} 4{ }^{+} \mathrm{CD} 25^{+}$regulatory $\mathrm{T}$ cells during early mycobacterial infection increases cytokine production but does not affect pathogen load. Immunol. Cell Biol. 84, 467-474 (2006).

26. Manca, C. et al. Virulence of a Mycobacterium tuberculosis clinical isolate in mice is determined by failure to induce Th1 type immunity and is associated with induction of IFN-alpha /beta. Proc. Natl Acad. Sci. USA 98, 5752-5757 (2001).

27. Gerosa, F. et al. Differential regulation of interleukin 12 and interleukin 23 production in human dendritic cells. J. Exp. Med. 205, 1447-1461 (2008) 
28. Gopal, R. et al. IL-23-dependent IL-17 drives Th1-cell responses following Mycobacterium bovis BCG vaccination. Eur. J. Immunol. 42, 364-373 (2012).

29. Pitt, J. M. et al. Blockade of IL-10 signaling during bacillus Calmette-Guerin vaccination enhances and sustains Th1, Th17, and innate lymphoid IFNgamma and IL-17 responses and increases protection to Mycobacterium tuberculosis infection. J. Immunol. 189, 4079-4087 (2012).

30. Ordway, D. J. et al. Mycobacterium bovis BCG-Mediated Protection against W-Beijing Strains of Mycobacterium tuberculosis Is Diminished Concomitant with the Emergence of Regulatory T Cells. Clin. Vaccine Immunol. 18, 1527-1535 (2011)

31. Gonzalez-Juarrero, M., Turner, J., Basaraba, R. J., Belisle, J. T. \& Orme, I. M. Florid pulmonary inflammatory responses in mice vaccinated with Antigen-85 pulsed dendritic cells and challenged by aerosol with Mycobacterium tuberculosis. Cell. Immunol. 220, 13-19 (2002).

32. McShane, H., Behboudi, S., Goonetilleke, N., Brookes, R. \& Hill, A. V. Protective immunity against Mycobacterium tuberculosis induced by dendritic cells pulsed with both CD8 $(+)$ - and CD4 $(+)$-T-cell epitopes from antigen 85A. Infect. Immun. 70, 1623-1626 (2002).

33. Koo, M. S. et al. Phosphodiesterase 4 inhibition reduces innate immunity and improves isoniazid clearance of Mycobacterium tuberculosis in the lungs of infected mice. PLoS ONE 6, e17091 (2011).

34. Sergio, C. A., Bertolini, T. B., Gembre, A. F., Prado, R. Q. \& Bonato, V. L. CD11c $(+)$ CD103 $(+)$ cells of Mycobacterium tuberculosis-infected C57BL/6 but not of $\mathrm{BALB} / \mathrm{c}$ mice induce a high frequency of interferon-gammaor interleukin-17-producing CD4(+ ) cells. Immunology 144, 574-586 (2015).

35. Zelante, T. et al. CD103(+) Dendritic Cells Control Th17 Cell Function in the Lung. Cell Rep. 12, 1789-1801 (2015).

36. Liu, H. et al. Structure-based programming of lymph-node targeting in molecular vaccines. Nature 507, 519-522 (2014).

37. Kayes, T. et al. Agonistic anti-CD40 induces thyrocyte proliferation and promotes thyroid autoimmunity by increasing CD40 expression on thyroid epithelial cells. J. Immunol. 190, 3928-3938 (2013).

38. Zippelius, A., Schreiner, J., Herzig, P. \& Muller, P. Induced PD-L1 expression mediates acquired resistance to agonistic anti-CD40 treatment. Cancer Immunol. Res. 3, 236-244 (2015).

39. Lindenstrom, T. et al. Vaccine-induced th 17 cells are maintained long-term postvaccination as a distinct and phenotypically stable memory subset. Infect. Immun. 80, 3533-3544 (2012).

40. Andersen, P. \& Smedegaard, B. CD4 $(+)$ T-cell subsets that mediate immunological memory to Mycobacterium tuberculosis infection in mice. Infect. Immun. 68, 621-629 (2000).

41. Feng, C. G. \& Britton, W. J. CD ${ }^{+}$and $\mathrm{CD} 8{ }^{+}$T cells mediate adoptive immunity to aerosol infection of Mycobacterium bovis bacillus CalmetteGuerin. J. Infect. Dis. 181, 1846-1849 (2000).

42. Kipnis, A., Irwin, S., Izzo, A. A., Basaraba, R. J. \& Orme, I. M. Memory T lymphocytes generated by Mycobacterium bovis BCG vaccination reside within a CD4 CD44lo CD62 Ligandhi population. Infect. Immun. 73, 7759-7764 (2005).

43. Mainali, E. S. \& McMurray, D. N. Protein deficiency induces alterations in the distribution of T-cell subsets in experimental pulmonary tuberculosis. Infect. Immun. 66, 927-931 (1998).

44. Sakai, S. et al. Cutting edge: control of Mycobacterium tuberculosis infection by a subset of lung parenchyma-homing CD4 T cells. J. Immunol. 192, 2965-2969 (2014).

45. Marcus, S. A., Steinberg, H. \& Talaat, A. M. Protection by novel vaccine candidates, Mycobacterium tuberculosis DeltamosR and DeltaechA7, against challenge with a Mycobacterium tuberculosis Beijing strain. Vaccine 33, 5633-5639 (2015).

46. Garly, M. L. et al. BCG scar and positive tuberculin reaction associated with reduced child mortality in West Africa. A non-specific beneficial effect of BCG? Vaccine 21, 2782-2790 (2003).

47. Buffen, K. et al. Autophagy controls BCG-induced trained immunity and the response to intravesical BCG therapy for bladder cancer. PLoS Pathog. 10, e1004485 (2014).

48. Kleinnijenhuis, J. et al. Bacille Calmette-Guerin induces NOD2-dependent nonspecific protection from reinfection via epigenetic reprogramming of monocytes. Proc. Natl Acad. Sci. USA 109, 17537-17542 (2012).

49. Demangel, C. et al. Stimulation of dendritic cells via CD40 enhances immune responses to Mycobacterium tuberculosis infection. Infect. Immun. 69, 2456-2461 (2001).

50. Stylianou, E. et al. Exploring the vaccine potential of Dec-205 targeting in Mycobacterium tuberculosis infection in mice. Vaccine 29, 2279-2286 (2011).

51. Silva-Sanchez, A. et al. ESAT-6 targeting to DEC205 ${ }^{+}$antigen presenting cells induces specific- $T$ cell responses against ESAT-6 and reduces pulmonary infection with virulent Mycobacterium tuberculosis. PLoS ONE 10, e0124828 (2015).
52. Antonelli, L. R. et al. Intranasal Poly-IC treatment exacerbates tuberculosis in mice through the pulmonary recruitment of a pathogen-permissive monocyte/ macrophage population. J. Clin. Invest. 120, 1674-1682 (2010).

53. Lai, R. et al. Restoration of innate immune activation accelerates Th1-cell priming and protection following pulmonary mycobacterial infection. Eur. J. Immunol. 44, 1375-1386 (2014).

54. Bode, C., Zhao, G., Steinhagen, F., Kinjo, T. \& Klinman, D. M. CpG DNA as a vaccine adjuvant. Expert. Rev. Vaccines 10, 499-511 (2011).

55. Zent, C. S. et al. Phase I clinical trial of CpG oligonucleotide 7909 (PF03512676) in patients with previously treated chronic lymphocytic leukemia. Leuk. Lymphoma 53, 211-217 (2012).

56. Vonderheide, R. H. \& Glennie, M. J. Agonistic CD40 antibodies and cancer therapy. Clin. Cancer Res. 19, 1035-1043 (2013).

57. Beauchamp, N. M., Yammani, R. D. \& Alexander-Miller, M. A. CD8 marks a subpopulation of lung-derived dendritic cells with differential responsiveness to viral infection and toll-like receptor stimulation. J. Virol. 86, 10640-10650 (2012).

58. Semmrich, M. et al. Directed antigen targeting in vivo identifies a role for $\mathrm{CD}_{103}{ }^{+}$dendritic cells in both tolerogenic and immunogenic $\mathrm{T}$-cell responses. Mucosal Immunol. 5, 150-160 (2012).

59. Fuertes, M. B. et al. Host type I IFN signals are required for antitumor CD8 ${ }^{+} \mathrm{T}$ cell responses through CD8 $\{\text { alpha }\}^{+}$dendritic cells. J. Exp. Med. 208, 2005-2016 (2011).

60. Hildner, K. et al. Batf3 deficiency reveals a critical role for CD8alpha ${ }^{+}$ dendritic cells in cytotoxic T cell immunity. Science 322, 1097-1100 (2008)

61. Ashok, D. et al. Cross-presenting dendritic cells are required for control of Leishmania major infection. Eur. J. Immunol. 44, 1422-1432 (2014).

62. del Rio, M. L., Rodriguez-Barbosa, J. I., Kremmer, E. \& Forster, R. CD103- and $\mathrm{CD} 03^{+}$bronchial lymph node dendritic cells are specialized in presenting and cross-presenting innocuous antigen to $\mathrm{CD}^{+}$and $\mathrm{CD}^{+} \mathrm{T}$ cells.

J. Immunol. 178, 6861-6866 (2007).

63. Furuhashi, K. et al. Mouse lung $\mathrm{CD} 103^{+}$and CD11bhigh dendritic cells preferentially induce distinct $\mathrm{CD} 4{ }^{+}$T-cell responses. Am. J. Respir. Cell Mol. Biol. 46, 165-172 (2012).

64. Souza-Fonseca-Guimaraes, F. et al. Interferon-gamma and granulocyte/ monocyte colony-stimulating factor production by natural killer cells involves different signaling pathways and the adaptor stimulator of interferon genes (STING). J. Biol. Chem. 288, 10715-10721 (2013).

65. Zhan, Y. et al. GM-CSF increases cross-presentation and CD103 expression by mouse CD8(+ ) spleen dendritic cells. Eur. J. Immunol. 41, 2585-2595 (2011).

66. Santosuosso, M., McCormick, S., Zhang, X., Zganiacz, A. \& Xing, Z. Intranasal boosting with an adenovirus-vectored vaccine markedly enhances protection by parenteral Mycobacterium bovis BCG immunization against pulmonary tuberculosis. Infect. Immun. 74, 4634-4643 (2006).

67. Skeiky, Y. A. et al. Differential immune responses and protective efficacy induced by components of a tuberculosis polyprotein vaccine, Mtb72F, delivered as naked DNA or recombinant protein. J. Immunol. 172, 7618-7628 (2004).

68. Hoang, T. et al. ESAT-6 (EsxA) and TB10.4 (EsxH) based vaccines for pre- and post-exposure tuberculosis vaccination. PLoS ONE 8, e80579 (2013).

69. Boehm, F. et al. Deletion of Foxp $3^{+}$regulatory $\mathrm{T}$ cells in genetically targeted mice supports development of intestinal inflammation. BMC Gastroenterol. 12, 97 (2012).

70. Hernandez-Novoa, B. et al. Immune responses to Pneumocystis murina are robust in healthy mice but largely absent in CD40 ligand-deficient mice. J. Leukoc. Biol. 84, 420-430 (2008).

\section{Acknowledgements}

This work was supported by Washington University in St Louis, National Institute of Health grant HL105427, AI127172 to S.A.K., by the Ragon Institute of MGH, MIT, and Harvard and the U.S. Army Research Office through the Institute for Soldier Nanotechnologies (contract W911NF-13-D-0001) to D.J.I., American Lung Association Senior Research Training Fellowship RT-30592, Department of Molecular Microbiology, Washington University St Louis, and Alexander and Gertrude Berg Fellowship to K.L.G. J.R.-M. was supported by funds of the Department of Medicine, University of Rochester, and U19 AI91036. We thank Sarah Squires for animal breeding, and Drs June Treerat, Racquel Domingo-Gonzalez for technical assistance. D.J.I. is an investigator of the Howard Hughes Medical Institute.

\section{Author contributions}

K.L.G., M.A., R.G., J.K.K. and S.A.K. designed the experiments; K.L.G., M.A., S.D., K.D.M., R.G., W.H. and J.R-M. performed the experiments; K.L.G., W.H., T.D.C., J.K.K., J.R-M., M.N.A. and S.A.K. performed analyses; T.D.C., D.J.I provided reagents; K.L.G., S.D. and S.A.K. wrote the paper; K.L.G., T.D.C., D.J.I., J.K.K., J.R-M. and S.A.K. edited the paper. S.A.K., D.J.I., J.R-M. provided funding. 


\section{Additional information}

Supplementary Information accompanies this paper at http://www.nature.com/ naturecommunications

Competing financial interests: The authors declare no competing financial interests.

Reprints and permission information is available online at http://npg.nature.com/ reprintsandpermissions/

How to cite this article: Griffiths, K. L. et al. Targeting dendritic cells to accelerate T-cell activation overcomes a bottleneck in tuberculosis vaccine efficacy. Nat. Commun. 7, 13894 doi: $10.1038 /$ ncomms13894 (2016).
Publisher's note: Springer Nature remains neutral with regard to jurisdictional claims in published maps and institutional affiliations.

(c) (i) This work is licensed under a Creative Commons Attribution 4.0 International License. The images or other third party material in this article are included in the article's Creative Commons license, unless indicated otherwise in the credit line; if the material is not included under the Creative Commons license, users will need to obtain permission from the license holder to reproduce the material. To view a copy of this license, visit http://creativecommons.org/licenses/by/4.0/

(C) The Author(s) 2016 